\title{
Microscopic theory of the jamming transition of harmonic spheres
}

\author{
Ludovic Berthier, ${ }^{1}$ Hugo Jacquin, ${ }^{2}$ and Francesco Zamponi ${ }^{3}$ \\ ${ }^{1}$ Laboratoire Charles Coulomb, UMR 5221, CNRS and Université Montpellier 2, Montpellier, France \\ ${ }^{2}$ Laboratoire Matière et Systèmes Complexes, UMR 705\%, CNRS and Université Paris Diderot - Paris 7, \\ 10 rue Alice Domon et Léonie Duquet, 75205 Paris cedex 13, France \\ ${ }^{3}$ Laboratoire de Physique Théorique, UMR 8549, CNRS and Ecole Normale Supérieure, \\ 24 Rue Lhomond, 75231 Paris Cedex 05, France
}

(Dated: November 5, 2018)

\begin{abstract}
We develop a microscopic theory to analyze the phase behaviour and compute correlation functions of dense assemblies of soft repulsive particles both at finite temperature, as in colloidal materials, and at vanishing temperature, a situation relevant for granular materials and emulsions. We use a mean-field statistical mechanical approach which combines elements of liquid state theory to replica calculations to obtain quantitative predictions for the location of phase boundaries, macroscopic thermodynamic properties and microstructure of the system. We focus in particular on the derivation of scaling properties emerging in the vicinity of the jamming transition occurring at large density and zero temperature. The new predictions we obtain for pair correlation functions near contact are tested using computer simulations. Our work also clarifies the conceptual nature of the jamming transition, and its relation to the phenomenon of the glass transition observed in atomic liquids.
\end{abstract}

PACS numbers: 05.20.Jj, 61.43.-j, 64.70.qd

\section{INTRODUCTION}

\section{A. From atoms to balls}

It is common practice in physics textbooks to represent various states of matter by replacing atomic or molecular arrangements by assemblies of spherical balls. The analogy goes much deeper, since simple theoretical models of spherical, repulsive particles are known to display phase transitions between states of matter that are actually observed in atomic systems. About 50 years ago, Bernal $[1,2]$ proposed a theory of the liquid state based on the intuition that dense liquids have a structure similar to dense assemblies of macroscopic grains, and performed with coworkers experiments to analyze the microstructure of steel ball bearings. Since Bernal's insightful work, a large number of detailed experiments and numerical simulations have been made to characterize the structure of dense assemblies of spherical particles, both for hard particles relevant for granular materials [3] and for soft particles relevant for emulsions and foams [4].

However, while the structure of dense atomic liquids is by now well understood using the framework of statistical mechanics [5], the problem of sphere packing proved much more difficult to solve $[6,7]$. Indeed, there are two major obstacles to be faced when approaching this problem. First, sphere packings are inherently nonequilibrium systems where thermal fluctuations play no role. This has for instance led researchers to extend concepts of statistical mechanics to specifically address athermal packings implementing ideas first put forward in particular by Edwards [8-11]. A second major difficulty is that one must deal with both fluid and solid disordered states of matter, which are poorly understood subjects even for atomic systems driven by thermal fluctuations. In partic- ular, while dense liquids are appropriately described by liquid state theory, viscous liquids and amorphous materials are still the focus of intense research [12]. Following Bernal's insight, it was suggested that the fluid to solid transition observed in athermal systems shares similarities with the glass transition of supercooled molecular fluids [13]. Since its publication, the "jamming phase diagram" has striked lively debates and a large literature seeking in particular to understand similarities and differences in parallel studies of both thermal and athermal amorphous media [14].

In this work, we are interested in describing theoretically fluid and solid disordered states of matter observed in dense assemblies of spherical repulsive particles both for finite temperatures relevant for colloidal materials, and in the athermal limit relevant for emulsions and foams. Unlike Bernal, we now have at our disposal theories for the liquid state, but this is not sufficient $[15,16]$. Liquid state theory is essentially based on perturbation theory extrapolating from the gas state, and for this reason it inherently fails to describe the phase transitions to low temperature and high density solid phases of matter. However, the liquid-crystal transition is conveniently described by density functional theory, where one starts from an approximated form for the free energy as functional of the local density profile and looks for a transition between uniform and periodically modulated minima of this functional. Early studies of the glass transition $[17,18]$ used density functional theory and looked for amorphous density profiles that minimize the free energy. Theoretical developments in the field of spin glasses [19-21] suggested similarities between supercooled liquids and a class of mean-field spin glass models which can be extensively analyzed theoretically $[22,23]$. These works gave birth to the so-called Random First 
Order Transition (RFOT) theory [21], which is currently one of the most consistent theories of the glass transition $[12,24-26]$. Along with these conceptual developments, RFOT theory has been turned into a quantitative computational scheme in a series of pioneering works by Monasson [27], Franz, Mézard and Parisi [28-31]. These works can schematically be seen as an extension of the tools developed in liquid state theory to study systems near the glass transition and the properties of amorphous solids, but they remain "mean-field" in nature in the sense that the glassy phase is assumed to be of the same nature as the one obtained from various mean-field perspectives [12]. We wish to emphasize, however, that "mean-field" has a very specific meaning in the context of glass theories. Although the theory we shall develop is a mean-field theory, it remains fully microscopic in nature (we start from the interaction between the particles), and it is able to capture many-body correlations between particles giving rise to a complex free energy landscape, which is crucial to accurately predict both the phase diagram of the system and correlation functions. In particular, we are able to provide a theoretical description of the distinct nature of both glass and jamming transitions.

Our goal is thus to apply concepts and tools from RFOT theory to analyze the glassy and jammed phases of systems of soft repulsive particles, thus contributing to better understanding amorphous, athermal states of matter [14, 32]. In previous work, Parisi and Zamponi (PZ) extended the scheme devised by Mézard and Parisi (MP) to treat systems of hard spheres [33]. The result is a RFOT theory of the glass transition and jamming of hard spheres; this theory is expected to be correct in the limit of large space dimension [33] and for a modified model where particles are randomly displaced over the box [34] (both limits being of a mean-field nature), and gives quantitatively correct results in three dimensions [33]. In a similar spirit, Mari et al. devised a model of hard spheres with diluted interactions [35] where "mean-field" replica calculations become exact, although the solution remains quite involved [36]. We wish to unify the approaches of MP and PZ to describe simultaneously the critical properties on both sides of the jamming transition occurring in athermal materials, and in its vicinity at finite temperatures. The theory that we develop in this work is, in our opinion, the equivalent, for the jamming transition, of a Landau mean-field theory for second order phase transition.

\section{B. Glass and jamming transitions in harmonic spheres}

A model system that can interpolate between finite temperature glasses and hard spheres is the model of harmonic spheres. An assembly of $N$ spherical particles of diameter $\sigma$ is enclosed in a volume $V$ in three spatial dimensions, interacting with a soft harmonic repulsion of

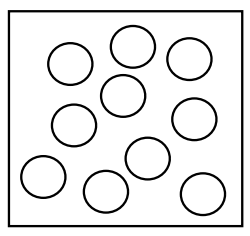

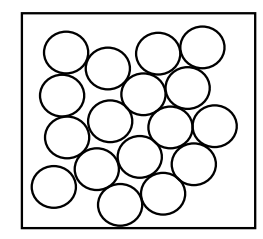

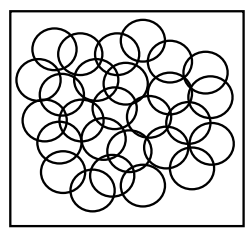

$\varphi_{\mathrm{j}}$
FIG. 1: The jamming transition of harmonic spheres at zero temperature. Below $\varphi_{j}$, particles do not overlap (as in hard sphere configurations) and the system flows. Above $\varphi_{j}$, there is a finite density of overlaps, and the energy and pressure are finite. In this region, hard spheres configurations cannot be found.

finite range:

$$
\phi(r)=\epsilon(1-r / \sigma)^{2} \theta(\sigma-r),
$$

where $r \geq 0$ is the interparticle distance, $\theta(r)$ is the Heaviside step function and $\epsilon$ controls the strength of the repulsion. The model (1), originally proposed to describe wet foams [37], has become a paradigm in numerical studies of the $T=0$ jamming transition $[32,38]$. It has also been studied at finite temperatures [39-41], and finds experimental realizations in emulsions, soft colloids and grains [42]. The model has the two needed control parameters to explore the jamming phase diagram: the temperature $T$ (expressed in units of $\epsilon$ ), and the fraction of the volume occupied by the particles in the absence of overlap, the volume fraction $\varphi=\pi N \sigma^{3} /(6 V)$. We now set $\sigma$ and $\epsilon$ to unity.

Over the last decade, a large number of numerical observations was reported for this model [14, 32]. A jamming transition is observed at $T=0$ at some critical volume fraction $\varphi_{j}$, the density above which the packings carry a finite density of particle overlaps. This transition is pictorially represented in Fig. 1. Numerically, the zero-temperature energy density, $e_{\mathrm{GS}}$, and pressure, $P$, are found to increase continuously from zero above $\varphi_{j}$ as power laws [38]. The pair correlation function of the density fluctuations [5], $g(r)$, develops singularities near $\varphi_{j}[43,44]$, which are smoothed by thermal excitations [41]. In particular, $g(1)=\infty$ at $\varphi_{j}$ and $T=0$. This behavior implies that the density of contacts between particles, $z$, jumps discontinuously from 0 to a finite value, $z_{j}$, at $\varphi_{j}$, and increases further algebraically above $\varphi_{j}[32,38]$. Thus, the jamming transition appears as a phase transition taking place in the absence of thermal motion, with a very peculiar critical behavior and physical consequences observable experimentally in a large number of materials. Note however that in the unjammed state below $\varphi_{j}$, the specific configurations sampled by the system might depend on the details of the preparation protocol. In the absence of fluctuations for instance $[45,46]$, the contact number is not vanishing even below the jamming point, because particles remain 


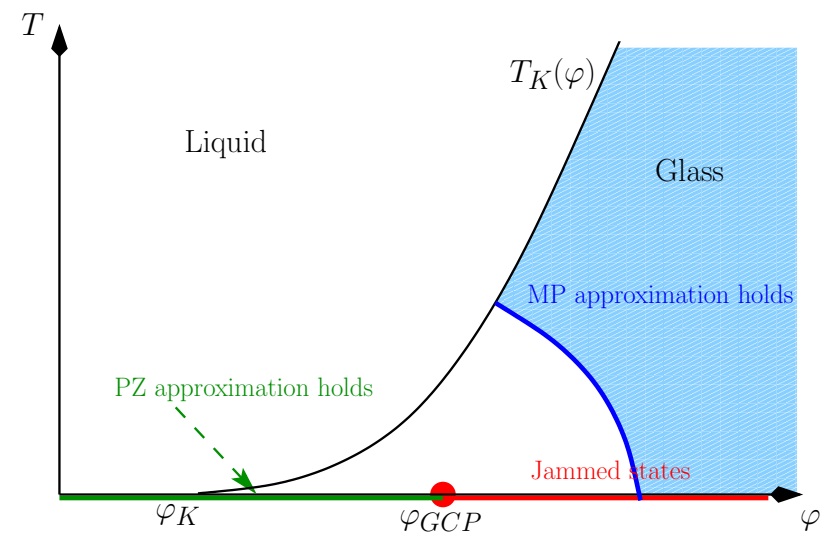

FIG. 2: (Color online) A schematic phase diagram for the glassy states of the model defined by Eq. (1) showing both the glass transition line at $T_{K}(\varphi)$ and the jamming point at $T=0$ and $\varphi=\varphi_{G C P}$. The Parisi-Zamponi (PZ) approach only holds at $T=0$ and $\varphi<\varphi_{G C P}$, while the Mézard-Parisi (MP) approach holds for large enough densities and temperatures. In this paper we develop a method to fill the gap between the $\mathrm{PZ}$ and MP analytical schemes to explore the vicinity of the jamming transition.

in contact and the left cartoon in Fig. 1 is not a faithful representation.

As discussed in detail by Krzakala and Kurchan [47], the jamming transition is very similar in nature to the so-called SAT-UNSAT transition of random constraint satisfaction problems $[48,49]$. These are problems where a large set of constraints has to be simultaneously satisfied. Upon increasing the density of constraints per variable, a phase transition is found, from a phase where the problem is typically solvable (SAT) to a phase where it is typically not (UNSAT). In the present context, the constraints are the non-overlapping conditions between spheres, and the control parameter is the density. At $\varphi_{j}$, the system goes from a phase where the non-overlap conditions can be satisfied to a phase where they cannot. In the context of random constraint satisfaction problems, it is very useful to introduce a soft version of the constraints, study the problem at finite temperature $T$, and finally take the $T \rightarrow 0$ limit $[50-52]$. The reason is that introducing a temperature allows to use powerful statistical mechanics tools, that can be used in a context where they are not a priori relevant [47].

To address the purely geometrical packing problem of soft spheres, we then suggest to study first the statistical mechanics of the model defined by Eq. (1) at finite temperatures, before taking the $T \rightarrow 0$ limit where jamming occurs. Based on the analogy with constraint satisfaction problems, we have in mind the schematic phase diagram reported in Fig. 2: see in particular the result for Potts glasses in [52], and see also [53]. In this picture, that we will derive in the following, the liquid undergoes a glass transition at some temperature $T_{K}(\varphi)$. The line $T_{K}(\varphi)$ vanishes at a volume fraction $\varphi_{K}$ which corresponds also to the ideal glass transition for hard spheres [33]. The point $\varphi_{K}$ is not the jamming transition: indeed, the ground state energy and pressure remain zero across $\varphi_{K}$. Above $\varphi_{K}$, the system enters at zero temperature a hard sphere glassy state. In this state, particles vibrate near well-defined (but random) positions, and the system is not yet jammed [33]. Jamming happens when the glass reaches its close packing density, which we shall call "glass close packing" (GCP) [33]. We identify the jamming transition with $\varphi_{G C P}$. In Fig. 2 the density interval $\left[\varphi_{K}, \varphi_{G C P}\right]$ is simply the amorphous analog of the interval $\varphi \in[0.54,0.74]$ for ordered states of hard spheres where a compressible crystalline structure exists at thermal equilibrium.

The main theoretical difficulty in the study of the jamming transition at $\varphi_{G C P}$ is that it happens deep inside the glass phase. Therefore, we need to develop first an accurate theoretical description of the glass phase. As discussed in detail in Ref. [16], previous theories of the glass phase fail (see Fig. 2). The MP approach holds only at high enough temperature or density, where a simple harmonic approximation for vibrations in the glass holds. The PZ approach holds only in the hard sphere region, $T=0$ and $\varphi<\varphi_{G C P}$. The central theoretical achievement that we report in this paper is an approximation scheme that can naturally interpolate between those of $\mathrm{MP}$ and $\mathrm{PZ}$ and is correct in the entire vicinity of the jamming transition, thus allowing us to fully explore the phase diagram of Fig. 2, and in particular the region of low temperature, $T \ll T_{K}(\varphi)$ and $\varphi \sim \varphi_{G C P}$. We obtain theoretically from a first principle calculation a large number of the observed behaviors of harmonic spheres, and predict new results for the correlation functions of this system around the jamming transition. A short report of our results has been published in Ref. [54].

\section{Discussion}

Our approach is very different from, but complementary to, recent theoretical works on jamming.

The aim of our work is to show, directly from the Hamiltonian, that the jamming transition exists, and determine from first principles its location and properties. Other approaches assume the existence of jammed states and try to obtain geometrical informations on them $[10,55]$. A particularly interesting approach is to develop a scaling picture of the jamming transition by showing that the transition is characterized by a diverging correlation length [56-58]. This approach allows to obtain a very detailed description of the jamming transition [59], that explains the anomalous scalings in shear [38,60] and transport [61] properties of amorphous jammed packings.

Moreover, our method is crucially based on equilibrium statistical mechanics. In the glassy phase, on approaching $T_{K}(\varphi)$ and below it, the equilibrium Gibbs distribution of the system is the superposition of many distinct ergodic components, each representing a differ- 
ent glassy state; each of these "basins" (see Appendix A for a more detailed discussion) is visited, in our theory, according to its equilibrium weight. On the contrary, most of the numerical works on jamming focus on specific dynamic protocols, that often are athermal and therefore always out of equilibrium. It has been shown that these protocols sample jammed states with highly nonuniform probabilities [46, 62]. Even thermal protocols (see e.g. [43]) inevitably fall out of equilibrium before $T_{K}(\varphi)$ is reached, and therefore produce jammed packings with probabilities that are strongly different from the equilibrium ones [33, 47]. Still, a crucial observation is that the critical properties around the (protocol dependent) jamming density $\varphi_{j}$ are largely independent of the protocol, and therefore of the probability with which jammed states are visited. This observation suggests that the critical properties are shared by almost all jammed states, and therefore an equilibrium sampling of these states can appropriately describe these properties, as we showed in this work. Of course, some care will have to be taken when comparing with some specific protocols, in which no fluctuations are present at all (neither thermal, nor mechanical) $[45,46]$. In those cases, as we already mentioned, the contact number is not vanishing even below the jamming point. Once these effects are taken into account, we believe that the generic picture we discuss in the following also applies to these protocols.

Finally, it is worth mentioning that if compression rates are small, partial crystallization takes place and the system can jam at any packing fraction between $\varphi_{G C P}$ and the close packing density corresponding to the most dense crystal [63-65]. In our theory, crystallization is not included by construction, as we will explain in the following, therefore this effect is absent. Extending this study to take into account partial crystallization would be interesting but probably very hard.

The outline of the paper is the following. In Sec. II, we present the approximation scheme developed earlier, and describe our own approximation specifically developed to study harmonic spheres. In Secs. III, IV and V respectively, we present results concerning the glass transition, the jamming transition and the correlation functions. In Sec. VI we compare the theoretical results with numerical data. We discuss and conclude the article in Sec. VII.

\section{THE METHOD}

\section{A. The complexity}

In mean-field models of glasses, upon decreasing temperature, a number $\mathcal{N}$ of metastable states, that scales as the exponential of $N$, appears [23]. The dynamics of the glass is slowed down considerably: once the glass has fallen into one of these metastable states, the barrier it has to cross in order to jump to another metastable state is proportional to $N$, due to the mean-field nature of the models. In mean-field theory there are two distinct tran- sitions upon lowering the temperature towards the glass state [23]: at a first transition $T_{d}$, the metastable states appear, giving rise to a finite complexity $\Sigma=N^{-1} \log \mathcal{N}$, which is the entropic contribution due to the exponential number of metastable states. Below this temperature, the dynamics of the system is arrested: this temperature is indeed identified with the dynamical transition predicted by Mode-Coupling Theory [20, 25]. At a lower temperature $T_{K}$, the complexity vanishes, and the number of metastable states is sub-exponential; this has been identified with the Kauzmann entropy crisis transition [66]. Therefore at the mean-field level the complexity is finite between $T_{d}$ and $T_{K}$. Although we will not discuss this physics in the rest of the paper, it is worth noting at this point that RFOT theory predicts that in a three (or finite) dimensional system the complexity is finite only locally. This is because the system gains entropy by accessing many different states; this creates an entropic driving force that allows the system to maintain its ergodicity by visiting all its metastable states. A nucleation theory of supercooled liquids has been developed based on these ideas [24-26], which predicts that the transition at $T_{d}$ is avoided in finite dimension through activated events, and the dynamics is arrested only at $T_{K}$. These ideas have been initially proposed on a phenomenological basis [24-26] but are now starting to be confirmed by renormalization group computations in simple models [67, 68]. While nucleation processes are surely important to describe activated dynamics below $T_{d}$, one expects that the local structural properties will not be affected by them and therefore mean-field theory should provide precise predictions as long as static and local observables are concerned. For this reason, in this work we follow Mézard and Parisi [28, 30] and directly apply the mean-field concepts described above to three dimensional glass-formers, neglecting activation.

Let us denote by $\mathcal{C}$ the microscopic configuration of the system. The starting point of the discussion is the assumption that in the glassy region, phase space can be partitioned in pure states, indexed by $\alpha=1, \cdots, \mathcal{N}$, in such a way that a given configuration $\mathcal{C}$ belongs to one state $\alpha$. A practical implementation of this construction, which has been studied recently in Ref. [69], is discussed in Appendix A. Under this decomposition, the partition function can be written as:

$$
Z=\sum_{\mathcal{C}} e^{-\beta E(\mathcal{C})}=\sum_{\alpha=1}^{\mathcal{N}} \sum_{\mathcal{C} \in \alpha} e^{-\beta E(\mathcal{C})}
$$

We define an effective free-energy of a pure state $f_{\alpha}$ by $f_{\alpha}=-\frac{1}{N \beta} \ln \left(\sum_{\mathcal{C} \in \alpha} e^{-\beta E(\mathcal{C})}\right)$, and the complexity $\Sigma$ as the logarithm of the number $\mathcal{N}(f)$ of pure states at a given free-energy $f: \Sigma(f)=\frac{1}{N} \log \mathcal{N}(f)$. We assume that, as in exactly solvable mean-field models [23], the complexity $\Sigma(f)$ is non-zero in a finite interval $\left[f_{\min }, f_{\max }\right]$ of free energy, and it vanishes linearly 
at $f_{\min }$. We find:

$$
Z=\int d f \mathcal{N}(f) e^{-\beta f}=\int d f e^{-N \beta(f-T \Sigma(f))} .
$$

It is convenient for the following of the paper to consider, instead of the total free energy $F=-T N^{-1} \log Z$, a "free entropy" $\mathcal{S}=N^{-1} \log Z=-\beta F$; the advantage is that this quantity has a finite limit for $T \rightarrow 0$, which is the entropy of hard spheres. Saddle-point evaluation at thermodynamic limit gives an expression for the free entropy:

$$
\begin{aligned}
& \mathcal{S}(T, \varphi) \equiv \frac{1}{N} \ln Z=\Sigma\left(f^{*}(T, \varphi)\right)-\beta f^{*}(T, \varphi), \\
& \frac{\partial \Sigma}{\partial f}\left(f^{*}(T, \varphi)\right)=\frac{1}{T} .
\end{aligned}
$$

The glass transition is reached at the Kauzmann temperature $T_{K}(\varphi)$, when the complexity vanishes:

$$
\Sigma\left(f^{*}\left(T_{K}(\varphi), \varphi\right)\right)=\Sigma\left(f_{\text {min }}\right)=0 .
$$

When $T<T_{K}(\varphi)$, the system has the free energy $f_{\min }(T, \varphi)$ and the complexity sticks to zero. Therefore, detecting the glass transition and calculating the free energy of the glass phase amounts to finding a way to compute the complexity as a function of the free energy.

A schematic plot of $T_{K}(\varphi)$ for harmonic spheres is reported in Fig. 2. Although our approach does not allow for a reliable calculation of $T_{d}(\varphi)$ [33], we expect that the latter will be slightly bigger than $T_{K}(\varphi)$ and follow a similar trend, as it can be shown in mean-field lattice models (see e.g. Fig.2 in Ref. [70]).

\section{B. Replicating the system}

The need to replicate the system in order to compute the thermodynamics of the glass originates from the difficulty in defining an order parameter for the glass transition. Indeed, below $T_{K}$ the system freezes in an amorphous density profile which is structurally very similar to a typical liquid configuration, therefore distinguishing the liquid and the glass by means of some observable related to structure (like a correlation function) is extremely difficult. Edwards and Anderson proposed a way out of this difficulty by introducing an order parameter defined as $[22]$

$$
q_{E A}=\frac{1}{V} \int d^{3} x\langle\delta \rho(x)\rangle^{2}
$$

where $\langle\delta \rho(x)\rangle=\langle\rho(x)\rangle-\rho$ is the thermodynamic average of the fluctuation of the local density $\rho(x)$ with respect to its spatial average $\rho=N / V$. This order parameter is clearly zero in the liquid phase while it is non-zero in a frozen amorphous phase. The problem is that its computation is not easy since one has to compute the local density and average its square. A solution is to introduce two identical copies of the system and compute

$$
q_{E A}=\frac{1}{V} \int d^{3} x\left\langle\delta \rho_{1}(x)\right\rangle\left\langle\delta \rho_{2}(x)\right\rangle
$$

The problem is that if the two replicas are independent, they will freeze in completely different profiles, and $q_{E A}$ will be zero since the integrand will have random signs. To avoid this problem, we must couple the two replicas via a small interaction, and then compute $q_{E A}$ by sending first $N \rightarrow \infty$ and then switching off the interaction. In a liquid phase, since ergodicity is maintained, the copy will eventually decorrelate from the original, and the order parameter will go to zero. In an ergodicity-broken phase, the copy will stay trapped near the original and the order parameter will go to a finite value. This prescription allows one to detect the glass transition but does not give any information on the free-energy of the glass and thus the glass phase itself.

Monasson [27] showed that replicas can provide a simple way to compute the complexity. He introduced an arbitrary number of replicas $m$, and assumed that a small coupling between each pair of replicas is present, in such a way that all replicas are constrained to be in the same metastable state. Since the number of metastable states does not change, the complexity is unaffected by this procedure. The partition function $Z_{m}$ of this replicated liquid then reads:

$$
Z_{m}=\int d f e^{-N \beta(m f-T \Sigma(f))} .
$$

The number $m$ of replicas is eventually taken to be noninteger, creating an additional $m$ dependence of the freeenergy and complexity of the glass. Equations (4) and (5) become now:

$$
\begin{aligned}
& \mathcal{S}(m ; T, \varphi)=\Sigma\left(f^{*}(m ; T, \varphi)\right)-m \beta f^{*}(m ; T, \varphi), \\
& \frac{\partial \Sigma}{\partial f}\left(f^{*}(m ; T, \varphi)\right)=\frac{m}{T} .
\end{aligned}
$$

This $m$-dependence of the free entropy allows one to compute the complexity as follows. We can determine $\Sigma$ and $f^{*}$ as functions of $m$ at fixed $(\varphi, T)$ using

$$
\begin{aligned}
& \Sigma=-m^{2} \frac{\partial(\mathcal{S} / m)}{\partial m} \\
& f^{*}=-T \frac{\partial \mathcal{S}}{\partial m} .
\end{aligned}
$$

Then one can eliminate parametrically the dependence on $m$ and in this way reconstruct the full curve $\Sigma(f)$.

We showed in the previous section that for $T<T_{K}$ the free energy of the glass is equal to $f_{\min }(T)$, the point where $\Sigma(f)=0$. Therefore, to compute $f_{\min }(T)$, we must impose that $\Sigma(f)=\frac{\partial(\mathcal{S} / m)}{\partial m}=0$, which is equivalent to finding the minimum of $\mathcal{S} / m$ with respect to $m$. Let us call $m^{*}(T, \varphi)$ the point where $\mathcal{S} / m$ assumes its minimum. 
Since $\Sigma=0$ at the minimum, using Eq. (10), we get $\mathcal{S}\left(m^{*} ; T, \varphi\right)=-m^{*} \beta f_{\min }(T, \varphi)$ which implies that:

$$
\begin{aligned}
\mathcal{S}_{\text {glass }}(T, \varphi) & =-\beta f_{\text {glass }}(T, \varphi)=-\beta f_{\text {min }}(T, \varphi) \\
& =\frac{\mathcal{S}\left(m^{*} ; T, \varphi\right)}{m^{*}(T, \varphi)}=\min _{m} \frac{\mathcal{S}(m ; T, \varphi)}{m} .
\end{aligned}
$$

Now we see that, in order to compute the thermodynamics of the ideal glass, one must be able to calculate the free entropy of a replicated liquid. In the following, we will present several approximation schemes that we applied and adapted, when necessary, for harmonic spheres.

\section{Effective potential approximation}

Computing the free-energy and complexity of the glass amounts to computing the free entropy of a $\mathrm{m}$ times replicated liquid. One can use a replicated version of standard liquid theory approximations such as the replicated Hyper-Netted-Chain (HNC) approximation [28, 29], but these kind of approximations usually break down deep in the glass phase. For a discussion of the reasons for the failure of replicated HNC, see Ref. [33]. One can also follow Refs. [30,31] and start at high density, and perform cage expansions: at high densities the copies stay close to the originals, forming molecules of size $A$. One can then expand the replicated free entropy with respect to this parameter $A$. However, to be able to explicitly compute the various integrals appearing in the computation, one traditionally has to suppose that the attraction between copies is harmonic, an assumption which breaks down for hard spheres, as discussed in [33]. To treat correctly the replicated hard sphere system, Parisi and Zamponi [33] developed an effective potential approximation, which in the end amounts to performing an expansion in powers of $\sqrt{A}$ instead of $A$. We explain in the following the extension of the effective potential approximation to finite temperature harmonic spheres. By construction, we will see that we are bound to recover both high density MP results and zero temperature PZ results in a unified treatment.

The starting point of all these approaches is the assumption that (due to the implicit coupling between replicas) the replicated system is composed of molecules made of $m$ atoms, each belonging to a different replica. Making use of standard theory of molecular liquids, one can express the free energy as a functional of the single molecule density and the interaction potential, see [33] for details. In order to make the computation tractable, one then makes a Gaussian ansatz for the probability distribution function of the positions $\bar{x}$ of the replicas within a molecule [31, 33]:

$$
\rho\left(\vec{x}_{1} \cdots \vec{x}_{m}\right)=\int d^{3} X \prod_{a=1}^{m} \frac{1}{(2 \pi A)^{3 / 2}} e^{-\frac{\left(\vec{x}_{a}-\vec{x}\right)^{2}}{2 A}}
$$

We can now integrate out all replicas except one. The effective interaction between particles in replica $1, \phi_{e f f}$, is

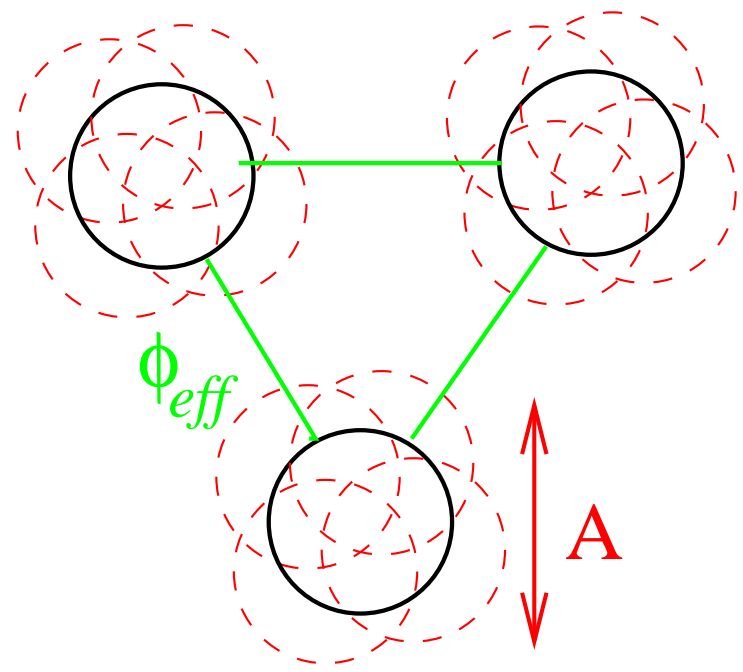

FIG. 3: (Color online) A schematic representation of the effective potential approximation. Each particle in the original liquid is replicated $m$ times (dashed spheres). Assuming that the replicated particles form a molecule of average cage size $A$, we trace out in the partition sum the degrees of freedom of $(m-1)$ copies of the liquid to obtain an effective onecomponent liquid (black spheres) interacting with an effective pair potential $\phi_{\text {eff }}$ (green lines).

obtained by averaging the full interaction over the probability distribution of the two molecules:

$$
\begin{aligned}
& e^{-\beta \phi_{e f f}\left(\vec{x}_{1}-\vec{y}_{1}\right)} \equiv e^{-\beta \phi\left(\vec{x}_{1}-\vec{y}_{1}\right)}\left\langle\prod_{a=2}^{m} e^{-\beta \phi\left(\vec{x}_{a}-\vec{y}_{a}\right)}\right\rangle_{\vec{x}_{1}, \vec{y}_{1}} \\
& =\int d^{3} x_{2} \cdots d^{3} x_{m} d^{3} y_{2} \cdots d^{3} y_{m} \times \\
& \times \rho\left(\vec{x}_{1} \cdots \vec{x}_{m}\right) \rho\left(\vec{y}_{1} \cdots \vec{y}_{m}\right) \prod_{a=1}^{m} e^{-\beta \phi\left(\vec{x}_{a}-\vec{y}_{a}\right)},
\end{aligned}
$$

We get after some simple manipulation:

$$
e^{-\beta \phi_{e f f}(r)}=e^{-\beta \phi(r)} \int d^{3} r^{\prime} \gamma_{2 A}\left(\vec{r}^{\prime}\right) q\left(A, T ; \vec{r}-\vec{r}^{\prime}\right)^{m-1},
$$

where $\gamma_{2 A}$ is a normalized and centered Gaussian of variance $2 A$ and $q(A, T ; r)=\int d^{3} r^{\prime} \gamma_{2 A}\left(\vec{r}^{\prime}\right) e^{-\beta \phi\left(\vec{r}-\vec{r}^{\prime}\right)}$ is a function that can be explicitly computed for our harmonic potential and is given in Eq. (B2) of Appendix B 1. This calculation is schematically represented in Fig. 3.

We can rewrite this result using bipolar coordinates:

$$
\begin{aligned}
& e^{-\beta \phi_{e f f}(r)}=e^{-\beta \phi(r)} \frac{1}{r \sqrt{4 \pi A}} \times \\
& \times \int_{0}^{\infty} d u u\left[e^{-\frac{(r-u)^{2}}{4 A}}-e^{-\frac{(r+u)^{2}}{4 A}}\right] q(A, T ; u)^{m-1} .
\end{aligned}
$$

The integration of replicas $2 \cdots m$ induces also threebody (and more generally many-body) interactions on 
replica 1; if we neglect these, and just keep the two-body effective potential, we obtain that the free entropy of the replicated liquid is the sum of an harmonic part $\mathcal{S}_{h}(m, A)$ plus the free energy of a liquid interacting via the effective potential: $\mathcal{S}=\mathcal{S}_{h}+\mathcal{S}_{\text {liq }}\left[\phi_{\text {eff }}\right]$ [33]. Furthermore, we define

$$
\begin{aligned}
& Q(r)=e^{-\beta\left(\phi_{e f f}(r)-m \phi(r)\right)}-1 \\
& e^{-\beta \phi_{\text {eff }}(r)}=e^{-\beta m \phi(r)}(1+Q(r))
\end{aligned}
$$

we note that $Q(r)$ is equal to zero when $A=0$, therefore for small $A$ we suppose that $Q(r)$ is a small perturbation, and we make use of equilibrium liquid perturbation theory. We finally obtain for the replicated free entropy $\mathcal{S}(m, A ; T, \varphi)=\frac{1}{N} \log Z_{m}$ :

$$
\begin{aligned}
\mathcal{S}(m, A ; T, \varphi) & =S_{h}(m, A)+\mathcal{S}_{l i q}(T / m, \varphi) \\
& +\frac{3 \varphi}{\pi} \int d^{3} r g_{l i q}(T / m, \varphi ; r) Q(r), \\
\mathcal{S}_{h}(m, A)= & \frac{3}{2}(m-1) \ln (2 \pi A)+\frac{3}{2}(m-1+\ln m),
\end{aligned}
$$

where $\mathcal{S}_{\text {liq }}(T, \varphi)=N^{-1} \log Z_{\text {liq }}$ is the free entropy of the liquid, $g_{l i q}(T, \varphi ; r)$ its correlation function (to be computed at temperature $T / m)$.

Equation (19) allows for a full calculation of the replicated free entropy, and gives back both the MP small cage expansion in powers of $A$ at high density, and the PZ expansion in powers of $\sqrt{A}$ at zero temperature. Details about this can be found in the Appendix B 2 and C. The main theoretical goal of the paper is now achieved [54]: we obtained a set of equations that allow us to make a connection between zero temperature hard spheres and finite temperature harmonic spheres. Note that the molecular liquid is assumed to be in a liquid state, therefore crystallization is excluded a priori from the theory [33].

\section{Low temperature approximation}

Our purpose in interpolating between zero and finite temperature was to be able to probe the vicinity of the jamming point in the $(\varphi, T)$ phase diagram, thus we are interested mainly in the low temperature behaviour of the glass. We can exploit this by making an approximation that will allow us to push the analytical calculation much further. Defining the cavity distribution function $y$ by $g(T, \varphi ; r)=e^{-\beta \phi(r)} y(T, \varphi ; r)$, we make the approximation of taking the cavity function of the liquid as a constant and equal to its $T=0, r=1$ value, which we call $y_{\text {liq }}^{H S}(\varphi)$. As $T$ goes to zero, we see that the exponential factor converges towards a step function around $r=1$, so that in all integrals that are cut at $r=1$ by the pair potential, we can safely evaluate $y$ at its $r=1$ value. Expanding $y$ in powers of $T$, it is easy to convince oneself that the temperature dependence leads to subdominant contributions in the integrals. Thus we suppose:

$$
g_{l i q}(T / m, \varphi ; r) \sim e^{-\beta m \phi(r)} y_{l i q}^{H S}(\varphi) .
$$

Plugging this approximation into Eq. (19), we get (the details of the computation are in Appendix B 3):

$$
\begin{aligned}
& \mathcal{S}(m, A ; T, \varphi)= S_{h}(m, A)+\mathcal{S}_{l i q}(T / m, \varphi) \\
&+4 \varphi y_{l i q}^{H S}(\varphi) G(m, A ; T) \\
& G(m, A ; T)=3 \int_{0}^{\infty} d r r^{2}\left[q(A, T ; r)^{m}-e^{-\beta m \phi(r)}\right],
\end{aligned}
$$

where $\mathcal{S}_{\text {liq }}(T / m, \varphi)$ is the free entropy of the nonreplicated liquid, evaluated at an effective temperature $T / m$. These are the finite-temperature version of the replicated free entropy of hard-spheres obtained in Ref. [33].

Many technical aspects presented in the following already appear in [33], apart from the existence of a new control parameter, the temperature. The average cage radius $A^{*}(m ; T, \varphi)$ is obtained by maximization of $\mathcal{S}$ against $A$. We find an implicit equation for $A^{*}(m ; T, \varphi)$ :

$$
\begin{aligned}
J\left(m, A^{*}(m ; T, \varphi) ; T\right) & =\frac{9}{4 \pi \rho y_{l i q}^{H S}(\varphi)}, \\
J(m, A ; T) & \equiv \frac{A}{1-m} \frac{\partial G(A, m ; T)}{\partial A} \\
& =3 \frac{m A}{1-m} \int_{0}^{\infty} d r r^{2} q(A, T ; r)^{m-1} \frac{\partial q(A, T ; r)}{\partial A} .
\end{aligned}
$$

\section{E. Liquid theory}

The last quantities that we need to compute are the free entropy $\mathcal{S}_{l i q}$ and the pair correlation function $g_{l i q}(r)$ of the liquid. Given the pair correlation function, which represents the probability of finding a particle at distance $r$ from a particle fixed at the origin, we can express the internal energy $U$ as:

$$
U(T, \varphi)=12 \varphi \int_{0}^{\infty} d r r^{2} g(T, \varphi ; r) \phi(r) .
$$

Plugging the low temperature approximation Eq. (21) for the liquid into this, we obtain:

$$
U_{l i q}(T, \varphi) \underset{T \rightarrow 0}{\sim} 12 \varphi y_{l i q}^{H S}(\varphi) \int_{0}^{1} d r r^{2}(1-r)^{2} e^{-\beta(1-r)^{2}}
$$

We are interested in computing the free entropy $\mathcal{S}_{l i q}$ of the liquid, which is related to the liquid free-energy $F_{l i q}$ by $\mathcal{S}_{l i q}(T, \varphi)=-\beta F_{l i q}(T, \varphi)$. The free entropy has a finite limit for hard spheres $T \rightarrow 0$, thus allowing to make the connection between hard spheres and soft spheres at finite temperature. Making use of the standard identity 
$U(T)=\frac{\partial(F / T)}{\partial(1 / T)}$, we can derive the low temperature approximation for the free entropy of the liquid:

$$
\begin{aligned}
& \mathcal{S}_{\text {liq }}(T, \varphi)=S_{\text {liq }}^{H S}(\varphi)+6 \varphi y_{\text {liq }}^{H S}(\varphi) \\
& \times\left[\frac{\sqrt{\pi}}{2} \sqrt{T}(2+T) \operatorname{erf}\left(\frac{1}{\sqrt{T}}\right)+T\left(e^{-1 / T}-2\right)\right],
\end{aligned}
$$

where $y_{l i q}^{H S}(\varphi)$ and $\mathcal{S}_{l i q}^{H S}(\varphi)$ are short-hand notations for $y_{l i q}(T=0, \varphi ; r=1)$ and $\mathcal{S}_{l i q}(T=0, \varphi)$.

At this level of approximation, it is clear that the only input that is needed from liquid theory is the equation of state of the hard sphere liquid. From any given equation of state one can easily deduce the hard sphere free entropy $\mathcal{S}_{l i q}^{H S}$ and cavity function $y_{l i q}^{H S}$. The most reasonable choice would be to use the phenomenological CarnahanStarling (CS) equation of state, as in [33], that provides the best fit to the hard sphere pressure.

In this paper we use instead the Hyper-Netted Chain (HNC) approximation: the HNC equations are non-linear integro-differential equations that require a numerical solution. Although $\mathrm{HNC}$ is known to be less accurate for the hard sphere system, using HNC allows us to also compare the present directly our results to the ones obtained from the MP approach valid at large density and finite temperatures. The procedure for solving these equations is described in full details in [71]. The HNC approximation overestimates $y_{l i q}^{H S}$ by $20 \%$ in the relevant range of volume fraction $\varphi \sim 0.64$. This has the effect of reducing the glass transition density obtained from the theory, from the value $\varphi_{K}=0.62$ obtained from CS [33] to $\varphi_{K}=0.58$ obtained with HNC. Therefore the reader should keep in mind that the glass densities reported in the following are lower than the correct ones. In any case, here we are more interested in the low-temperature scaling in the glass phase than to the actual value of the glass transition density. We also checked that the scaling results are insensitive to the precise choice of the equation of state of the hard sphere liquid.

\section{THERMODYNAMICS OF THE GLASS}

\section{A. Complexity and phase diagram}

As discussed above, the glass transition is signalled by the point where the saddle point in Eq. (3) reaches the minimum $f_{\min }$ at which the complexity $\Sigma(f)$ vanishes. In the replica formalism, the equilibrium complexity $\Sigma\left(f^{*}\right)$ in Eq. (6) corresponds to the complexity in Eq. (12) evaluated at $m=1$. We call this quantity the "equilibrium" complexity of the liquid $\Sigma_{e q}(T, \varphi)$. The latter is easily

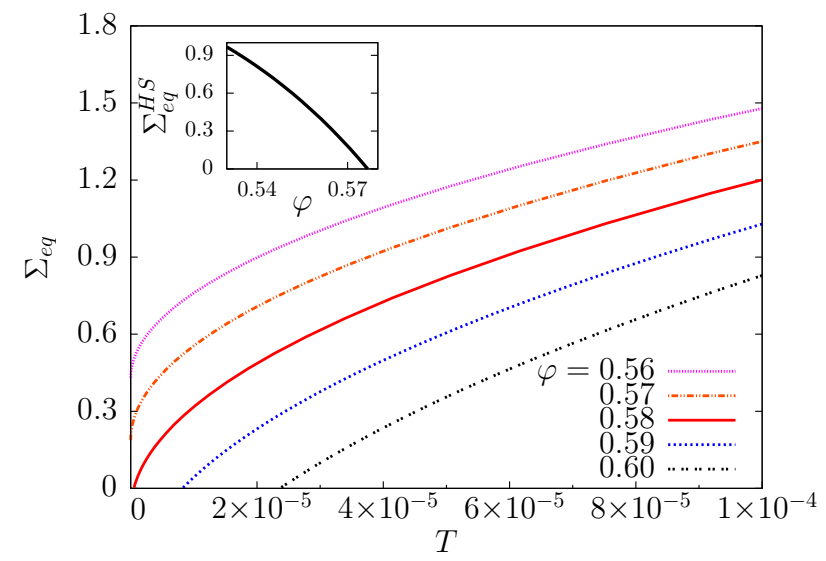

FIG. 4: (Color online) Equilibrium complexity against temperature for several volume fractions around $\varphi_{K}$, calculated within the effective potential approximation. Inset: complexity of hard spheres, obtained as the $T \rightarrow 0$ limit of the complexity $\Sigma_{e q}$, plotted against the volume fraction

computed by expanding the equations around $m=1$ :

$$
\begin{aligned}
\Sigma_{e q}(T, \varphi)= & S_{l i q}(T, \varphi)-\frac{3}{2} \ln \left(2 \pi A^{*}(m=1 ; T, \varphi)-3\right. \\
-12 \varphi y_{l i q}^{H S}(\varphi) H\left(m=1, A^{*}(m=1 ; T, \varphi) ; T\right), & \\
H(m, A ; T) \equiv & \frac{1}{m} \frac{\partial G(m, A ; T)}{\partial m} \\
= & \frac{1}{m} \int_{0}^{\infty} d r r^{2} q(A, T ; r)^{m} \ln q(A, T ; r) \\
& -\frac{\beta}{m} \int_{0}^{1} r^{2} \phi(r) e^{-m \beta \phi(r)} .
\end{aligned}
$$

We see that the only unknown in the previous equation is the optimal cage radius $A^{*}$. It is computed with Eq. (24) evaluated at $m=1$.

Since our computation makes connection with the hard spheres calculation of $\mathrm{PZ}$, we expect to retrieve all their results in the $T=0$ limit for $\varphi<\varphi_{G C P}$. Thus for $\varphi$ smaller than $\varphi_{K}$ (the Kauzmann transition of hard spheres), the system should be in a liquid phase, and thus its complexity should not vanish, and converge to the hard sphere complexity $\Sigma_{e q}^{H S}(\varphi)$ found by PZ. In practice, since PZ performed a $\sqrt{A}$ expansion, whereas we did not, our numerical values for $\varphi_{K}$ and $\Sigma_{e q}^{H S}(\varphi)$ could differ slightly. Nevertheless, $A$ is typically found between $10^{-3}$ and $10^{-6}$, so that the difference is indeed very small. For example $\varphi_{K}$ calculated by PZ differs from our value by less than $6 \cdot 10^{-3}$.

For $\varphi \geq \varphi_{K}$, the complexity vanishes at a temperature $T_{K}(\varphi)$, called the Kauzmann temperature, that increases with the density. These results are summarized in Fig. 4, where we show the complexity as a function of temperature for several volume fractions around $\varphi_{K}$. In the inset we show the zero temperature limit of the complexity $\Sigma_{e q}^{H S}$ as a function of the density, that vanishes at $\varphi_{K}$. 


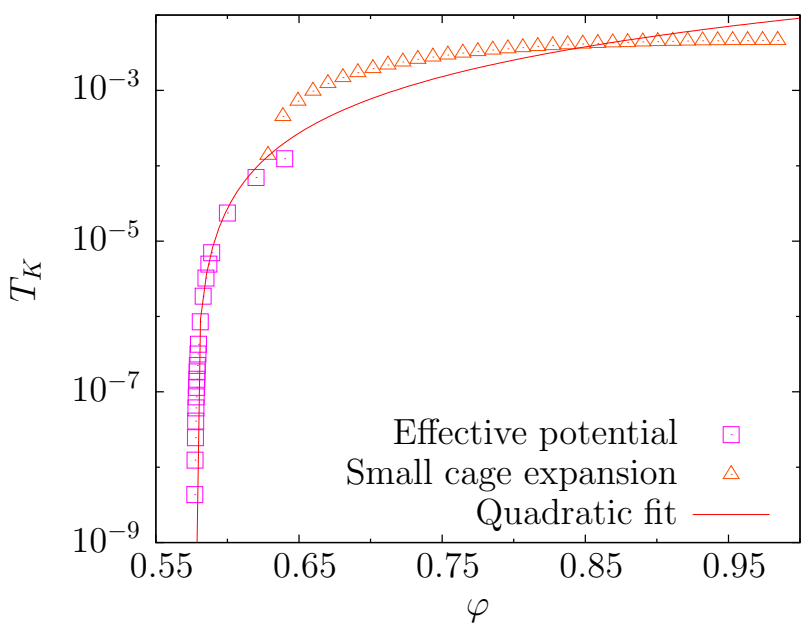

FIG. 5: (Color online) Kauzmann temperature against volume fraction, within the the effective potential approach developed in this work (open squares). The quadratic fit (red line) gives an estimated Kauzmann transition for hard spheres at $\varphi_{K}=0.576898$. The small cage Mézard-Parisi approach breaks down at low temperatures and low densities, as explained in Ref. [16].

From the complexity we deduce the phase diagram shown in Fig. 5. In this figure we report $T_{K}(\varphi)$, as obtained in the framework of the effective potential approximation developed in this paper, and we compare it with the result obtained from the Mézard-Parisi small cage expansion [16]. In the effective potential case we find that the Kauzmann temperature goes to zero at $\varphi_{K} \approx 0.5769$, quadratically in $\varphi-\varphi_{K}$. Conversely, the result from the small cage expansion is a finite $T_{K}(\varphi)$ which jumps to zero abruptly at a value of $\varphi$ which is unrelated to hard sphere results. This is due to fact that the small cage expansion is valid only in the region indicated in Fig. 2. On the other hand, our effective potential computation becomes inaccurate when the temperature is too high because of the approximation in Eq. (21). Still we obtain a reasonable matching of the two approximation schemes for intermediate densities around $\varphi=0.64$. It would be easy, in principle, to reconcile the two approximations at all volume fractions, including the crossover regime, by avoiding the low temperature approximation made in Eq. (21), but these computations would require a much heavier numerical treatment.

\section{B. Relaxation time and glass fragility}

The calculation of the liquid complexity, apart from signalling the glass transition, can be related to the relaxation time of the liquid by making use of the AdamGibbs scenario [72-74], which relates the relaxation time $\tau$ of a liquid to its configurational entropy. In the case of our mean-field vision of the phase space of the liquid, the configurational entropy can be identified with the equi-

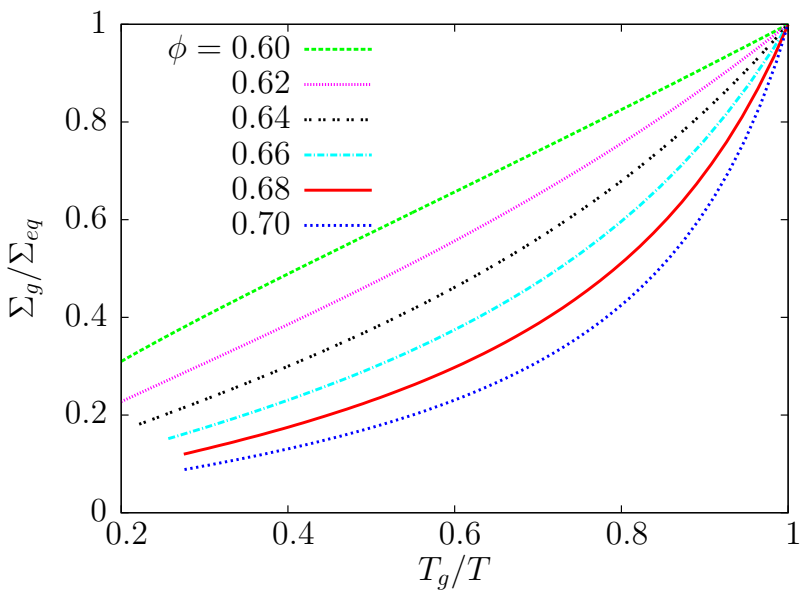

FIG. 6: (Color online) A thermodynamic Angell plot of $\Sigma_{g} / \Sigma_{e q}$, with $\Sigma_{g}=1$ as a definition of the glass transition, plotted against $T_{g} / T$, for different volume fractions. The thermodynamic fragility is predicted to increase rapidly with volume fraction, in agreement with numerical simulations [39].

librium complexity $\Sigma_{e q}$. In that case the Adam-Gibbs relation yields:

$$
\tau(T, \varphi)=A \exp \left(\frac{c}{T \Sigma_{e q}(T, \varphi)}\right)
$$

where $c$ is a constant. We can push the correspondence between complexity and relaxation time a bit further, and use it to extract informations on the fragility of the glass. The fragility of a glass quantifies how different the relaxation processes are from usual Arrhenius relaxation processes, and can be observed by representing the logarithm of the relaxation time against the inverse temperature. A linear curve is then the signature of an Arrhenius relaxation, while steeper curves indicate a greater fragility, i.e. a faster increase of the relaxation time.

In Refs. [39, 40], Berthier and Witten showed that, for harmonic spheres, compressing the system leads to a significant increase of the fragility. Using our results for the complexity, we are able to check qualitatively whether replica theory can reproduce this feature. Indeed, thanks to Eq. (31), the fragility can be extracted equivalently from the relaxation time or from the complexity. Since the fragility is usually evaluated at the conventionally defined laboratory glass transition, we arbitrarily define the glass transition temperature $T_{g}(\varphi)$ as the temperature at which the equilibrium complexity is equal to one, $\Sigma\left(T_{g}(\varphi), \varphi\right)=1$, which is a typical value of the configurational entropy at the glass transition in most numerical simulations and experiments (its precise value is immaterial for our purposes). Using these values of $T_{g}$ and $\Sigma_{g}=1$, we can construct an Angell plot for the complexity following Ref. [75]. In Fig. 6 we show the inverse of the complexity, linked to the logarithm of the relaxation time by Eq. (31), plotted against $T_{g} / T$, for several densities. The fragility is the slope of the curves in $T_{g} / T=1$ [75]. One can clearly see that increasing 


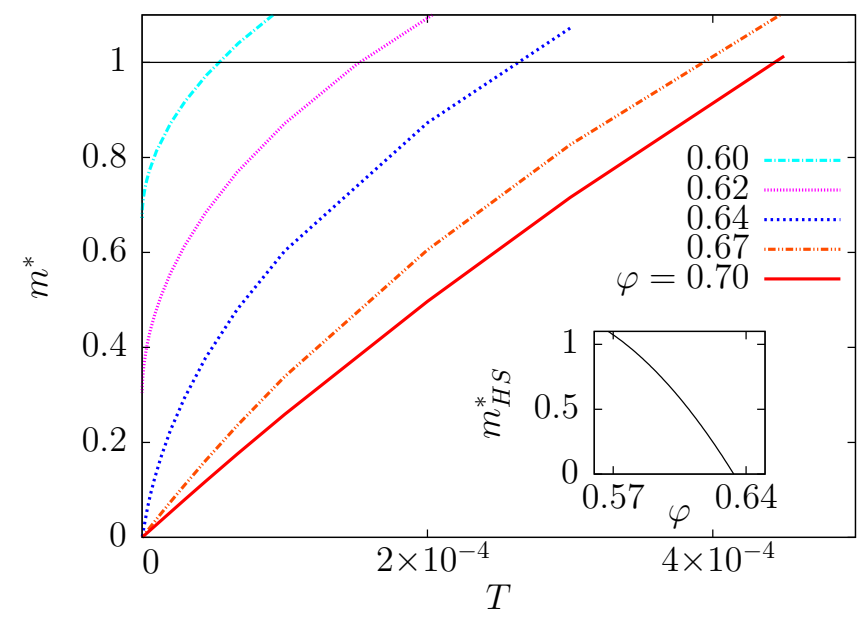

FIG. 7: (Color online) Optimal number of replicas $m^{*}$ as a function of $T$, for several volume fractions. The inset shows the $T \rightarrow 0$ limit of $m^{*}$ as a function of the volume fraction.

the density drastically increases the fragility of the glassformer.

\section{Free-energy of the glass}

We turn now to the calculation of the free entropy of the glass, $\mathcal{S}_{\text {glass }}$. We have seen in Sec. II that in order to compute $\mathcal{S}_{\text {glass }}(T, \varphi)$, one needs to optimize the replicated free entropy $\mathcal{S}(m, A ; T, \varphi) / m$ defined in Eq. (22) with respect to $A$ first, via Eq. (24), then with respect to $m$, via Eq. (13). Calling $A^{*}(T, \varphi)$ and $m^{*}(T, \varphi)$ the optimal values of $A$ and $m$, we obtain:

$$
\mathcal{S}_{\text {glass }}(T, \varphi)=\frac{\mathcal{S}\left(m^{*}(T, \varphi), A^{*}(T, \varphi) ; T, \varphi\right)}{m^{*}(T, \varphi)} .
$$

Starting from Eq. (32), we can deduce all quantities of interest, for instance the pressure and energy of the glass and its specific heat. The derivations are reported in Appendix B 4. In particular, our approximations imply that the energy of the glass $U_{\text {glass }}$ can be computed directly from the knowledge of the effective potential $\phi_{\text {eff }}$ via the following relation:

$$
U_{\text {glass }}(T, \varphi)=12 \varphi y_{\text {liq }}^{H S}(\varphi) \int_{0}^{1} d r r^{2}(1-r)^{2} e^{-\beta \phi_{e f f}(r)} .
$$

Similarly, we find that the reduced pressure (or "compressibility factor") $p=\beta P / \rho$ is given by

$$
\begin{aligned}
& p_{\text {glass }}(T, \varphi)=\frac{1}{m^{*}} p_{\text {liq }}\left(T / m^{*}, \varphi\right) \\
&-\frac{4 \varphi}{m^{*}}\left[y_{l i q}^{H S}(\varphi)+\varphi \frac{d y_{l i q}^{H S}(\varphi)}{d \varphi}\right] G\left(m^{*}, A^{*} ; T\right) .
\end{aligned}
$$

In Fig. 7, we show the behavior of the optimal number of replicas $m^{*}$ as a function of the temperature, for different volume fractions. We see that the $T \rightarrow 0$ limit of $m^{*}(T, \varphi)$ converges when $\varphi$ is not too large to a finite value, which we call $m_{H S}^{*}(\varphi)$, and which is shown in the inset. The replica parameter of hard spheres vanishes at a density $\varphi_{G C P}$, the glass close packing. This point is the equivalent, in our mean-field picture, of the jamming point of harmonic spheres. The behavior of the cage radius $A^{*}$ is similar to that of $m^{*}$. We will study in greater detail the behavior of the system around $\varphi_{G C P}$ in the next section.

From the knowledge of $m^{*}$ and $A^{*}$, we can deduce the energy and specific heat of the glass. We show in Fig. 8 the temperature evolution of the specific heat for three densities, one below $\varphi_{G C P}$, one very close to it, and one above. The temperature dependence of the specific heat is qualitatively in good agreement with the numerical data shown in Ref. [39], the only difference being that the abrupt jump predicted at $T_{K}$ using the theory is replaced by a smoother evolution and a small maximum at the numerical glass temperature.

Upon crossing the ideal glass transition, the specific heat undergoes a finite jump. We find that the amplitude of this jump increases continuously with $\varphi$ from $\varphi_{K}$. A qualitatively similar result was obtained in numerical simulations [39], where the jump of specific heat was studied at the numerical glass transition temperature. The behavior of the specific heat correlates well with the evolution of the thermodynamic fragility discussed in Fig. 6. Finally, we note that the $T \rightarrow 0$ limit of the specific heat jumps discontinuously from 0 to $3 / 2$ at $\varphi_{G C P}$, which reveals that the ground state properties of the glass phase change abruptly at the jamming transition, as we now study in more detail.

\section{JAMMING POINT OF HARMONIC SPHERES}

We now turn to the study of the region of the phase diagram deep in the glass phase, inside the line $T_{K}(\varphi)$ and close to the jamming point $\varphi_{G C P}$, see Fig. 2 . In this region, $m^{*}(T, \varphi)$ is very small, as we discussed in the last section (see Fig. 7). In principle, one could just compute $m^{*}(T, \varphi)$ and then take the limit $T \rightarrow 0$ and $\varphi \rightarrow \varphi_{G C P}$ ("jamming limit"). However, both numerically and analytically, it is much more convenient to exchange the optimization with respect to $m$ and $A$ with the jamming limit, take the latter first, and then optimize the free energy. This is because, in the jamming limit, many of the integrals that appear in the free energy simplify considerably. However, the scaling of $m$ and $A$ in the jamming limit is different depending on the order of the limits, which emphasizes the asymmetry that exists between both sides of the jamming point. In this section we discuss how to exchange the jamming limit with the optimization procedure. 


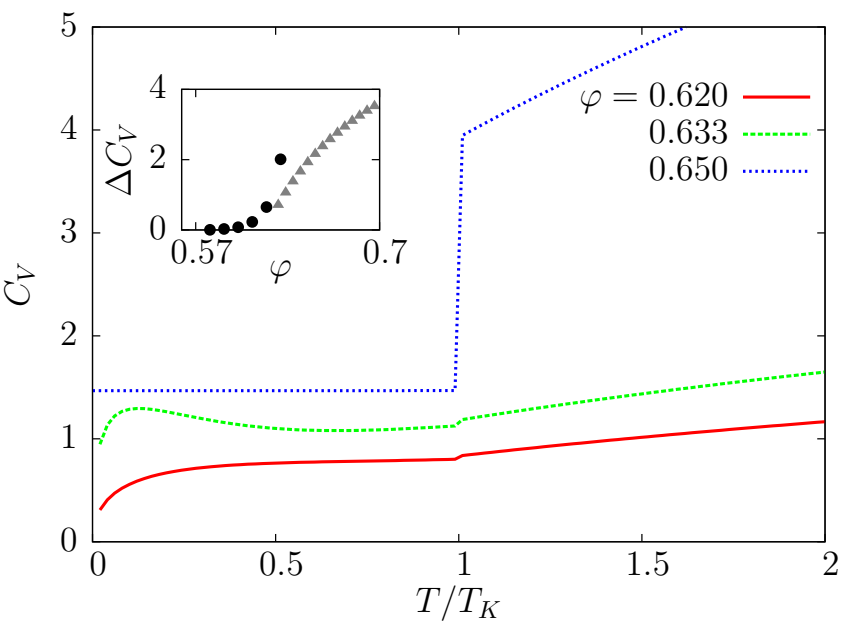

FIG. 8: (Color online) Specific heat $C_{V}$ of the system plotted against $T / T_{K}$, for several volume fractions. The curves for $\varphi=0.620,0.633$ are obtained with the effective potential method developed in this paper. The curve for $\varphi=0.650$ is obtained with the Mézard-Parisi small cage expansion [31], since at this density $T_{K}(\varphi)$ is too high and the effective potential method is not reliable. Inset: Specific heat jump at $T_{K}$; the black dots are obtained with the effective potential approximation, the gray triangles are obtained with the Mézard-Parisi small cage expansion.

\section{A. Zero temperature, below close packing}

We consider first the limit $T \rightarrow 0$ for fixed $\varphi<\varphi_{G C P}$. In this case, we are bound to recover the results of [33] for hard spheres. Thus, we expect the optimal number of replicas $m^{*}(T, \varphi)$ and cage radius $A^{*}(T, \varphi)$ to tend to finite values $m_{H S}^{*}(\varphi)$ and $A_{H S}^{*}(\varphi)$ when $T \rightarrow 0$. Therefore, to recover hard spheres results, one has to take the limit $T \rightarrow 0$ at fixed $A$ and $m$, and optimize the resulting free entropy over $A$ and $m$. The corresponding expression for the replicated free entropy is given in Appendix $\mathrm{C}$ and coincides with that of [33]. We refer to this paper for further details on the hard sphere case.

We solved numerically the optimization equations; the result for $m_{H S}^{*}(\varphi)$ is plotted in the inset of Fig. 7. Approaching $\varphi_{G C P}$ by the left, $m_{H S}^{*}$ tends to zero linearly:

$$
m_{H S}^{*}=\widetilde{\mu}\left(\varphi_{G C P}-\varphi\right),
$$

and $A_{H S}^{*}$ also goes to zero with $m$ as $\alpha m$. Thus the close packing limit for hard spheres can be computed by taking first the limit $T \rightarrow 0$, and then $m \rightarrow 0$ with $A=\alpha m$. Optimization on $A$ will now become an optimization on $\alpha$. From the inset of Fig. 7 we find

$$
\varphi_{G C P}=0.633353,
$$

and $\widetilde{\mu}=20.7487$, see Appendix $\mathrm{E}$ for more details. Therefore, we find that the location of the jamming transition is different from the density of the hard sphere glass transition, which confirms the distinct nature of both phenomena.

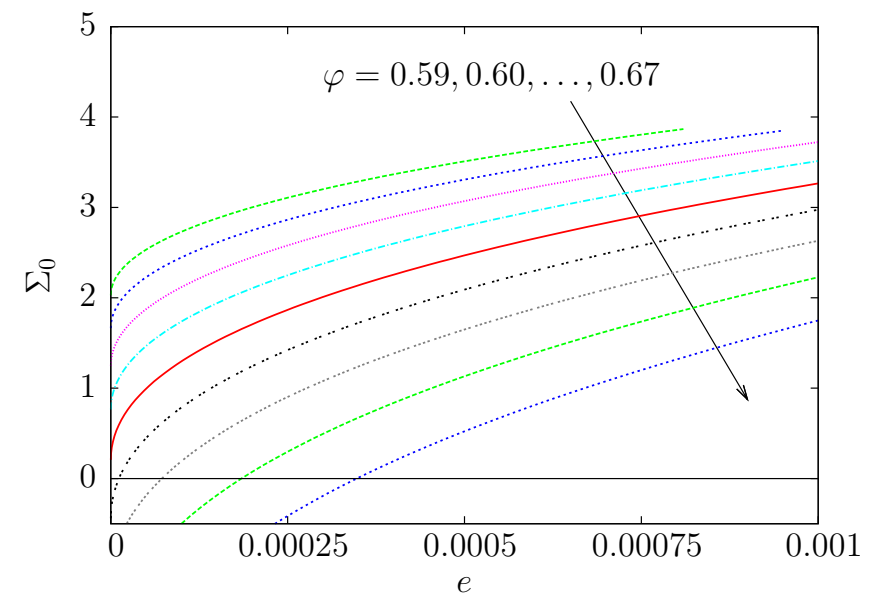

FIG. 9: (Color online) Complexity of the energy minima $\Sigma_{0}(e, \varphi)$ as a function of energy for several densities.

In this limit ( $T \rightarrow 0$ at finite $m$ and $A$ ), it is easy to see that $\phi_{\text {eff }}$ has an hard-core of the same size of the original potential. Therefore, from Eq. (33), it is straightforward to see that the energy of the glass vanishes: the system becomes indeed a system of hard spheres. From Eq. (34), since both $p_{\text {liq }}(T=0, \varphi)$ and $G(m, A ; T=0)$ are finite, we find that the reduced pressure goes to a finite value $p_{\text {glass }}(T=0, \varphi)$ which is the reduced pressure of the hard sphere glass. The latter diverges at $\varphi_{G C P}$ since $m^{*} \rightarrow 0$ at that point. One can show that for $\varphi \rightarrow \varphi_{G C P}^{-}$, since $m^{*}$ vanishes linearly, one obtains [33]

$$
p_{\text {glass }}(T=0, \varphi) \sim \frac{3.03430 \varphi_{G C P}}{\varphi_{G C P}-\varphi}
$$

where the prefactor is only $1 \%$ different from the correct value which is the space dimension $d=3$, predicted by free volume theory [76] and by the small cage expansion of Ref. [33].

\section{B. Zero temperature, above close packing}

For $\varphi>\varphi_{G C P}$, the harmonic spheres have a finite energy at $T=0$, due to overlaps. Then, because of the repulsion the spheres are jammed, and $A^{*}$ vanishes at $T=0$. It turns out from the numerical calculation of $m^{*}$ and $A^{*}$, both in the small cage expansion [31] and in the potential approximation (see the previous section) that the optimal number of replicas $m^{*}$ tends to zero when $T \rightarrow 0$. One finds that $m=T / \tau$ and $A=m \alpha$ with constants $\alpha$ and $\tau$. Therefore for $\varphi>\varphi_{G C P}$ one has to take the $T \rightarrow 0$ limit with $\alpha=A / m$ and $\tau=T / m$ kept constant. Optimization over $m$ and $A$ is replaced by an optimization over $\alpha$ and $\tau$.

The replicated free entropy (22) simplifies considerably in this limit. The details of the calculations are discussed in Appendix D. The expression for $\mathcal{S}_{0}(\alpha, \tau ; \varphi)$ in this limit is given in Eq. (D4). We first calculate the optimal 
cage radius $\alpha$ with Eq. (D5), and replace the solution $\alpha^{*}(\varphi)$ in Eq. (D4). We obtain the replicated free entropy as a function of $\tau, \mathcal{S}_{0}(\tau ; \varphi)=\mathcal{S}_{0}\left(\alpha^{*}, \tau ; \varphi\right)$. To understand the physical meaning of this quantity, we have to go back to Eq. (10) and take the limit $T \rightarrow 0$ with $m=T / \tau$. Since the free energy $f^{*}$ reduces to the energy $e^{*}$ for $T=0$, we get

$$
\mathcal{S}_{0}(\tau ; \varphi)=\lim _{T \rightarrow 0} \mathcal{S}(T / \tau ; T, \varphi)=\Sigma_{0}\left(e^{*}\right)-e^{*} / \tau .
$$

Following the reasoning that led to Eq. (12), we get

$$
\begin{aligned}
& \Sigma_{0}(\tau, \varphi)=\frac{\partial\left(\tau \mathcal{S}_{0}\right)}{\partial \tau}, \\
& e(\tau, \varphi)=\tau^{2} \frac{\partial \mathcal{S}_{0}}{\partial \tau},
\end{aligned}
$$

and therefore

$$
\begin{aligned}
e_{G S}(\varphi) & =U_{\text {glass }}(T=0, \varphi)=-\min _{\tau}\left[\tau \mathcal{S}_{0}\left(\alpha^{*}, \tau ; \varphi\right)\right] \\
& =-\tau^{*}(\varphi) \mathcal{S}_{0}\left(\alpha^{*}, \tau^{*}(\varphi) ; \varphi\right),
\end{aligned}
$$

since the ideal glass energy coincides with the amorphous ground state energy. We obtain the scaling of $\tau^{*}$ and of the energy around $\varphi_{G C P}$ by means of a development around $\varphi_{G C P}$. The calculation is presented in Appendix D, and the result is:

$$
\begin{aligned}
& \tau^{*}(\varphi) \sim \widetilde{\tau}\left(\varphi-\varphi_{G C P}\right), \\
& e_{G S}(\varphi) \sim \widetilde{e}\left(\varphi-\varphi_{G C P}\right)^{2},
\end{aligned}
$$

for $\varphi \rightarrow \varphi_{G C P}^{+}$, so that the jamming limit corresponds to $\tau \rightarrow 0$, as expected. We get $\varphi_{G C P}=0.633353$, $\widetilde{\tau}=0.00835535$ and $\widetilde{e}=0.263017$ (see Appendix E). In addition to the scalings in Eq. (41), we obtain the following scaling for the complexity against the ground-state energy for small $e$ :

$$
\Sigma_{0}(e, \varphi)=\Sigma_{0}^{H S}(\varphi)+A(\varphi) \sqrt{e}+\cdots
$$

The function $\Sigma_{0}(e, \varphi)$ is reported in Fig. 9. The energy of the ground state $e_{G S}(\varphi)$ corresponds to the vanishing of the complexity. We know that $\Sigma_{0}^{H S}(\varphi) \sim \varphi_{G C P}-$ $\varphi$. Since the coefficient $A$ is constant around $\varphi_{G C P}$, one obtains the quadratic scaling of the energy.

Finally, from Eq. (34) we see that the pressure $P_{\text {glass }}(T=0, \varphi)=\lim _{T \rightarrow 0}\left(\rho T p_{\text {glass }}\right)$ is proportional to $\tau^{*}$ and therefore

$$
P(\varphi) \sim \widetilde{P}\left(\varphi-\varphi_{G C P}\right)
$$

with $\widetilde{P}=0.403001$ (see Appendix E). This result is indeed consistent with the exact relation $P_{\text {glass }}(T=$ $0, \varphi)=\frac{6 \varphi^{2}}{\pi} \frac{d e_{G S}}{d \varphi}$.

In this section we have thus derived the scaling laws (41) and (43) first observed numerically above the jamming transition at zero temperature [38, 60], which indicate that solidity emerges continuously at the jamming density. These results also confirm the correspondance between the jamming transition observed numerically and the glass close packing density defined within our theoretical approach.

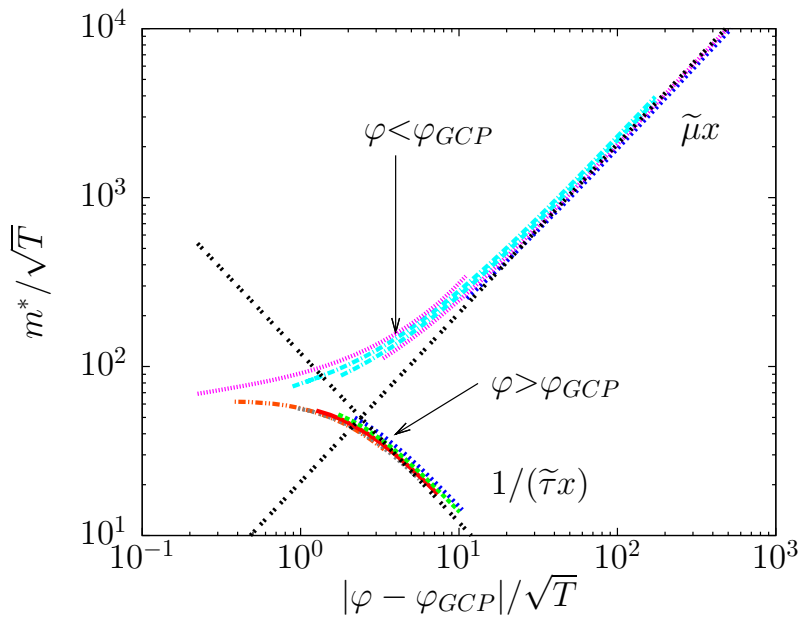

FIG. 10: (Color online) Scaling of $m^{*}(T, \varphi) / \sqrt{T}$ as a function of the rescaled temperature $\left|\varphi-\varphi_{G C P}\right| / \sqrt{T}$ for $\varphi=$ $0.58,0.59, \ldots, 0.70$. The asymptotic forms corresponding to Eq. (45) are also reported.

\section{Scaling around jamming}

We have shown in Secs. IV A and IV B that all the thermodynamic quantities are singular at $\varphi_{G C P}$ and $T=$ 0 ; for instance $m^{*}$ is finite below $\varphi_{G C P}$ while it vanishes proportionally to $T$ above $\varphi_{G C P}$. The reduced pressure is also finite below $\varphi_{G C P}$, while it diverges at $\varphi_{G C P}$ and is formally infinite above $\varphi_{G C P}$. Both the energy and the pressure vanish below $\varphi_{G C P}$ while they are finite above $\varphi_{G C P}$.

From this observation, and since all quantities are analytic at finite $T$, it follows that all these quantities must satisfy scaling relations if $T$ is small enough and $\varphi$ is close enough to $\varphi_{G C P}$ [77]. We discuss explicitly the case of $m^{*}$ for which we assume a scaling relation of the form

$$
m^{*}(T, \varphi)=T^{\gamma} \widetilde{m}_{ \pm}\left(\frac{\left|\varphi-\varphi_{G C P}\right|}{T^{\nu}}\right),
$$

where the two scaling functions correspond to the two sides of the transition. In the hard sphere limit $T \rightarrow 0$ and $\varphi<\varphi_{G C P}$, to recover Eq. (35) we need $\widetilde{m}_{-}(x \rightarrow$ $\infty)=\widetilde{\mu} x$ and $\gamma=\nu$. For $\varphi>\varphi_{G C P}$ instead, $m^{*}=$ $T / \tau^{*}(\varphi)$ and to recover Eq. (41) we need $\widetilde{m}_{+}(x \rightarrow \infty)=$ $1 /(\widetilde{\tau} x)$ and $\gamma=\nu=1 / 2$. Finally, we are led to the scaling

$$
\begin{aligned}
& m^{*}(T, \varphi)=\sqrt{T} \widetilde{m}_{ \pm}\left(\frac{\left|\varphi-\varphi_{G C P}\right|}{\sqrt{T}}\right), \\
& \widetilde{m}_{-}(x \rightarrow \infty)=\widetilde{\mu} x \\
& \widetilde{m}_{+}(x \rightarrow \infty)=\frac{1}{\widetilde{\tau} x} .
\end{aligned}
$$

This scaling is successfully tested in Fig. 10.

Qualitatively similar scaling forms (with different exponents) apply to thermodynamic quantities such as the 
energy, the pressure, or the complexity. Although we do not show the corresponding scaling plots explicitly, the energy and the pressure are plotted and compared with numerical data in the following (Fig. 12). Note finally that a scaling plot for the complexity is related, in the spirit of the Adam-Gibbs relation in Eq. (31), to the scaling plot of the numerically measured relaxation time reported in Ref. [39, 40].

\section{THE PAIR CORRELATION FUNCTION}

The pair correlation function is a key quantity that gives a lot of insight into the microstructure of dense fluids or packings. In this section we derive an expression for the correlation function of the glass and use it to discuss the scaling of the contact peak of $g(r)$ close to the jamming transition.

\section{A. General expression of the correlation function of the glass}

It has been shown in [33, Eqs.(67)-(68)] that one can compute the correlation function of the glass starting from the replicated free energy. This is done by adding a small perturbation $v(r)$ to the potential of replica 1 and taking the derivative of the free energy with respect to $v(r)$. The glass correlation function is then calculated as:

$$
g_{\text {glass }}(r)=-\frac{2}{\rho} \frac{\delta \mathcal{S}}{\beta \delta v(r)} .
$$

We start from Eq. (19) and we use approximation (21). Since the perturbation acts only on replica 1 , we have

$$
\begin{gathered}
\mathcal{S}(m, A ; T, \varphi)=S_{h}(m, A)+\mathcal{S}_{l i q}\left[\varphi, e^{-\beta m \phi(r)-\beta v(r)}\right] \\
+\frac{3 \varphi}{\pi} y_{l i q}^{H S}(\varphi) \int d^{3} r e^{-\beta m \phi(r)-\beta v(r)} Q(r),
\end{gathered}
$$

and $Q(r)$ depends only on the potentials of replicas 2 to $m$, so it is independent of $v$. Taking the derivative with respect to $v(r)$ we have, using again Eq. (21):

$$
\begin{aligned}
g_{\text {glass }}(r) & =g_{\text {liq }}(T / m, \varphi ; r)+y_{\text {liq }}^{H S}(\varphi) e^{-\beta m \phi(r)} Q(r) \\
& =y_{\text {liq }}^{H S}(\varphi) e^{-\beta m \phi(r)}[1+Q(r)] \\
& =y_{\text {liq }}^{H S}(\varphi) e^{-\beta \phi_{\text {eff }}(r)} .
\end{aligned}
$$

Therefore the correlation function of the glass is directly related to the effective potential within the low temperature approximation we used to compute the thermodynamics of the system. Of course, an improved expression for this correlation could be obtained by using the HNC approximation to describe the effective liquid. In that case, $g_{\text {glass }}(r)$ would be the HNC correlation of the effective liquid. Still, the much simpler expression (48) is enough to capture the scaling of $g_{\text {glass }}(r)$ around jamming, at least close to the first peak. In the rest of this section we discuss the scaling form that is obtained starting from Eq. (48).

A very interesting quantity related to $g(r)$ is the number of contacts, defined as the number overlaps per particle. Within our low temperature approximation it is given by:

$$
\begin{aligned}
z(T, \varphi) & =24 \varphi \int_{0}^{1} d r r^{2} g_{\text {glass }}(r) \\
& =24 \varphi y_{\text {liq }}^{H S}(\varphi) \int_{0}^{1} d r r^{2} e^{-\beta \phi_{e f f}(r)} .
\end{aligned}
$$

Using the definition of the effective potential, Eq. (15), we can rewrite this in a simpler way:

$z(T, \varphi)=24 \varphi y_{l i q}^{H S}(\varphi) \int_{0}^{\infty} d u u^{2} q(A, T ; u)^{m-1} \widehat{q}(A, T ; u)$,

with a function $\widehat{q}(A, T ; u)$ which is very similar to $q(A, T ; u)$; its form and the details of the derivation can be found in Appendix B 5 .

The starting point of the analysis of the correlation function of the glass are Eq. (48) and Eq. (17) where the effective potential is defined. In the following we keep the notation $e^{-\beta \phi_{\text {eff }}}$ since this function has a finite limit for $T \rightarrow 0$ even if $\beta$ is formally infinite.

\section{B. Scaling of the peak of the pair correlation at finite temperature}

Here we discuss the effect of thermal fluctuations on the maximum of $g_{\text {glass }}(r)$ near the the $T=0$ jamming transition at $\varphi_{G C P}$. This situation was studied numerically and experimentally in Refs. [15, 41]. We focus on the scaling of this maximum in a region of small $T$ and for $\varphi \sim \varphi_{G C P}$. In Fig. 11 we show the behavior of the maximum of $g_{\text {glass }}(r)$, which we call $g_{\max }$, as a function of the density, for temperatures ranging from $10^{-5}$ to 0 . This figure demonstrates that we are able to derive analytically all behaviors reported in Refs. [41, 43, 44, 78]. Namely, the density at which the pair correlation function reaches its maximum shifts towards higher values when the temperature departs from 0 and increases as $\sqrt{T}$, while the maximum $g_{\max }$ diverges as $\left|\varphi-\varphi_{G C P}\right|^{-1}$ on both sides of the transition.

As discussed in the previous section, the zero temperature limit is very different for $\varphi<\varphi_{G C P}$ or $\varphi>\varphi_{G C P}$. Thus we now discuss these two limits separately.

\section{Zero temperature below jamming: hard spheres}

In this case we send $T \rightarrow 0$ before taking the jamming limit $m \rightarrow 0$ and $A=\alpha m$, corresponding to hard spheres approaching jamming from below. Taking the limit $T \rightarrow$ 


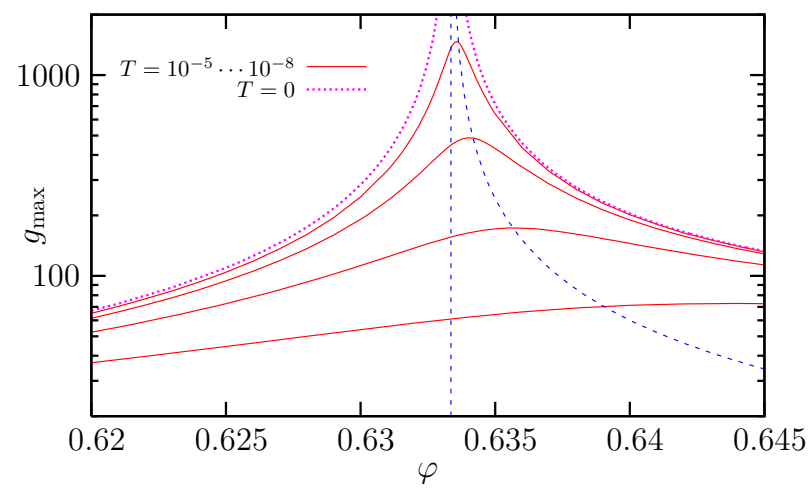

FIG. 11: (Color online) Evolution of the maximum of the glass pair correlation function with $T$ and $\varphi$. While $g_{\max }$ diverges on both sides of the transition at $T=0$ as $g_{\max } \sim$ $\left|\varphi-\varphi_{G C P}\right|^{-1}$, this divergence becomes a smooth maximum at finite $T$ near the transition, whose position shifts with temperature. This behavior compares very well with numerical $[41,43,44]$ and experimental observations [41, 78].

0 we get the expression of [33]:

$$
\begin{aligned}
e^{-\beta \phi_{e f f}(r)=} & \theta(r-1) \frac{1}{r \sqrt{4 \pi A}} \\
& \times \int_{0}^{\infty} d u u\left[e^{-\frac{(r-u)^{2}}{4 A}}-e^{-\frac{(r+u)^{2}}{4 A}}\right] q(A ; u)^{m-1},
\end{aligned}
$$

with $q(A ; r)$ defined in (C1). In the jamming limit $m \rightarrow 0$ and $A=\alpha m$ one can show (see Appendix C 3) that this has the scaling [33] in the region $r=1^{+}$:

$$
e^{-\beta \phi_{e f f}(r)}=\frac{1}{m} \Delta_{\alpha}\left(\frac{r-1}{m \sqrt{4 \alpha}}\right)
$$

where the function $\Delta_{\alpha}$ is given in Eq. (C16) and is very well approximated by $\Delta_{0}(\lambda)=1-\sqrt{\pi} \lambda e^{\lambda^{2}}(1-\operatorname{erf}(\lambda))$. For $\varphi \rightarrow \varphi_{G C P}^{-}$one has $m \sim \widetilde{\mu}\left(\varphi_{G C P}-\varphi\right)$ and $A^{*}=\alpha^{*} m$ with a finite $\alpha^{*}=\alpha^{*}\left(\varphi_{G C P}\right)$ : then the peak of the correlation function for $r=1^{+}$has a simple scaling form:

$$
g_{\text {glass }}(r) \sim \frac{y_{\text {liq }}^{H S}(\varphi)}{\widetilde{\mu}\left(\varphi_{G C P}-\varphi\right)} \Delta_{\alpha^{*}}\left[\frac{r-1}{\sqrt{4 \alpha^{*}} \widetilde{\mu}\left(\varphi_{G C P}-\varphi\right)}\right]
$$

the numerical values of the parameters can be found in Appendix E. The height of the peak scales as $g_{\text {glass }}\left(r=1^{+}, \varphi \rightarrow \varphi_{G C P}\right) \sim 1.09922 /\left(\varphi_{G C P}-\varphi\right)$ and its width as $\varphi_{G C P}-\varphi$. One can obtain scaling functions:

$$
\begin{aligned}
& g_{\text {glass }}(r)\left(\varphi_{G C P}-\varphi\right)=f\left[\frac{r-1}{\varphi_{G C P}-\varphi}\right], \\
& \text { with } f(\lambda)=\frac{y_{l i q}^{H S}(\varphi)}{\widetilde{\mu}} \Delta_{\alpha^{*}}\left[\frac{\lambda}{\widetilde{\mu} \sqrt{4 \alpha^{*}}}\right],
\end{aligned}
$$

or alternatively:

$$
\begin{aligned}
& \frac{g_{\text {glass }}(r)}{g_{\text {glass }}\left(1^{+}\right)}=f\left[(r-1)\left(1+4 \varphi g_{\text {glass }}\left(1^{+}\right)\right)\right], \\
& \text {with } f(\lambda)=\Delta_{\alpha^{*}}\left[\frac{\lambda}{8 \varphi y_{\text {liq }}^{H S}(\varphi) \sqrt{\alpha^{*}}}\right] .
\end{aligned}
$$

The number of contacts is the integral of the peak at $r=1^{+}$which is given by

$$
\begin{aligned}
z & \sim 24 \varphi \int_{\text {peak }} d r g_{\text {glass }}(r) \\
& \sim 24 \varphi y_{\text {liq }}^{H S}(\varphi) \sqrt{4 \alpha^{*}} \int_{0}^{\infty} d \lambda \Delta_{\alpha^{*}}(\lambda)
\end{aligned}
$$

Replacing the values given in Appendix E, we get $z=6.13720$ for $\varphi=\varphi_{G C P}$, which is slightly above the expected $z=2 d=6$.

Note that it has been shown in [33] that within a systematic expansion in $\sqrt{\alpha}$, one gets $z=2 d$ at first order. Here instead, in order to match with the soft sphere computation, we are using an approximation scheme which is not a consistent expansion in $\sqrt{\alpha}$. This could explain the fact that $z \neq 2 d$. It would be interesting to have a full computation of the next term in the small $\alpha$ expansion to check whether the equality $z=2 d$ survives at this order. Unfortunately this seems to be a quite hard task.

\section{Zero temperature above jamming}

For $\varphi>\varphi_{G C P}$, the appropriate limit is to send $T \rightarrow 0$ with $m=T / \tau$ and $A=\alpha m$. Starting from Eq. (D13) and taking this limit, the effective potential takes the following form (the calculation is presented in Appendix D 3):

$$
\begin{gathered}
e^{-\beta \phi_{e f f}(r)}=\theta(r-1)+\theta(1-r) \theta\left(r-\frac{4 \alpha}{\tau+4 \alpha}\right) \\
\times(1+4 \alpha / \tau)\left(1+\frac{4 \alpha(r-1)}{\tau r}\right)^{2} e^{-\frac{\tau+4 \alpha}{\tau^{2}}(r-1)^{2}} .
\end{gathered}
$$

Again, since $\tau \sim \varphi-\varphi_{G C P}$, close to $\varphi_{G C P}$ this function develops a delta peak close to $r=1$ with height $\left(\varphi-\varphi_{G C P}\right)^{-1}$ and width $\varphi-\varphi_{G C P}$. The main difference we observe with the hard sphere limit is that, despite the fact that both cases give rise to a delta function at $r=1$, the mechanism is different since for hard spheres the support is concentrated on $r=1^{+}$while for soft spheres it is concentrated on $r=1^{-}$. Another interesting observation is that the theory predicts that $g_{\text {glass }}(r)$ should vanish exactly for $r<\frac{4 \alpha}{\tau+4 \alpha}$; this means that no pair of spheres can have an overlap larger than $\frac{\tau}{\tau+4 \alpha}$. Indeed in the limit $\tau \rightarrow 0$ we recover the $G C P$ at which no overlaps are present. Unfortunately, a direct numerical test of this predicion is impossible: this is because when $1-r=\frac{\tau}{\tau+4 \alpha}$, the exponential term in Eq. (59) is equal to $\exp (-1 /(\tau+4 \alpha))$. Since both $\tau$ and $\alpha$ are already 
extremely small (of the order of $10^{-3}$ at best), $g_{\text {glass }}(r)$ is extremely small in the region where we predict it to vanish, and numerically the signal to noise ratio becomes too large in that region.

Again in the limit $\varphi \rightarrow \varphi_{G C P}^{+}$we obtain the scaling forms

$$
\begin{aligned}
& g_{\text {glass }}(r)\left(\varphi-\varphi_{G C P}\right)=f\left[\frac{1-r}{\varphi-\varphi_{G C P}}\right], \\
& f(\lambda)=\frac{y_{l i q}^{H S}(\varphi) 4 \alpha^{*}}{\widetilde{\tau}}\left(1-\frac{4 \alpha^{*} \lambda}{\widetilde{\tau}}\right)^{2} e^{-\frac{4 \alpha^{*} \lambda^{2}}{\widetilde{\tau}^{2}}},
\end{aligned}
$$

or alternatively:

$$
\begin{aligned}
& \frac{g_{\text {glass }}(r)}{g_{\text {glass }}\left(1^{+}\right)}=f\left[(r-1)\left(1+4 \varphi g_{\text {glass }}\left(1^{+}\right)\right)\right] \\
& f(\lambda)=\left(1-\frac{\lambda}{4 \varphi y_{l i q}^{H S}(\varphi)}\right)^{2} e^{-\frac{\lambda^{2}}{64 \alpha^{*}\left(\varphi y_{l i q}^{H S}(\varphi)\right)^{2}}} .
\end{aligned}
$$

The number of contacts is easily obtained from Eq. (49) and Eq. (59) by a change of variable:

$$
\begin{aligned}
z_{0}(\varphi) & =z(T=0, \varphi)=24 \varphi y_{l i q}^{H S}(\varphi) \int_{0}^{1} d r r^{2} e^{-\beta \phi_{e f f}(r)} \\
& =24 \varphi y_{l i q}^{H S}(\varphi) \int_{0}^{1} d r r^{2} e^{-\frac{(r-1)^{2}}{\tau+4 \alpha^{*}}} \\
& =8 \varphi y_{l i q}^{H S}(\varphi) G_{0}^{H S}\left(\alpha^{*}+\tau / 4\right),
\end{aligned}
$$

with $G_{0}^{H S}(\alpha)$ given in Eq. $(\mathrm{C} 7)$. The expression above is linear in $\tau$ at small $\tau$. Therefore, for $\varphi \rightarrow \varphi_{G C P}^{+}$, since $\tau \sim\left(\varphi-\varphi_{G C P}\right)$, we have $z_{0}(\varphi)=z_{0}\left(\varphi_{G C P}\right)+C(\varphi-$ $\left.\varphi_{G C P}\right)$. This result is at odds with the numerical finding of a square root behavior $[43,44]$. We comment further on this discrepancy in Sec. VII. At $\varphi_{G C P}$, we have

$$
z_{0}\left(\varphi_{G C P}\right)=8 \varphi y_{l i q}^{H S}(\varphi) G_{0}^{H S}(\alpha+\tau / 4)=6.13720,
$$

which is differs slightly from the expected isostatic value $z=2 d$, for the technical reasons already discussed above in the hard sphere case.

Note that this value of $z_{0}\left(\varphi_{G C P}\right)$ is identical to the one obtained on the hard sphere side (as it can be easily proven analytically), despite the fact that the two definitions of $z$ are not equivalent. In the soft sphere case we defined $z$ as the number of overlaps. On the hard sphere side, this number is strictly zero, therefore we defined $z$ as the integral of the delta peak at $r=1^{+}$.

\section{SCALING AROUND JAMMING: COMPARISON WITH NUMERICAL DATA}

In this section, we compare the prediction of the theory with numerical data, in particular to test the scaling in temperature and in $\delta \varphi=\varphi-\varphi_{G C P}$ around the glass close packing point.

\section{A. Details of the numerical procedure}

We investigated a system of $N=8000$ identical harmonic spheres. The system was prepared using the standard procedure of Ref. [38]. We started from a random configuration at high density; we then minimized the energy, and then reduced slowly the density, at each step minimizing the energy again, until a jammed configuration of zero energy and volume fraction $\varphi_{j}$ was found. This jammed configuration corresponds, according to the mean-field interpretation of [33], to the $T=0, p \rightarrow \infty$ limit of a given hard sphere glass, or equivalently to the $T \rightarrow 0, P \rightarrow 0$ limit of a corresponding soft sphere glass. Starting from that configuration, we prepared configurations at different $T$ and $\varphi$ by using molecular dynamics simulations as in Ref. [40] to thermalize the system inside the glass state selected by the initial jammed configuration. Since we always used very small $T$ and $\varphi \sim \varphi_{j}$, the system is not able to escape from that glass state, so it always remains in the vicinity of the original jammed configuration. This is a crucial requirement to numerically obtain scaling behavior near $\varphi_{j}$ as microscopic rearrangments would directly affect the location of $\varphi_{j}$ in the simulations [79]. We tested the absence of such an effect in our simulations by repeating the energy minimization starting from some equilibrated configurations at different $T$ and $\varphi$, and checking that we always found the same value of $\varphi_{j}$ (within numerical errors). For the particular series of run described below, we estimated $\varphi_{j}=0.643152 \pm 0.000020$ by fitting the zero temperature energy using $e(\varphi) \propto\left(\varphi-\varphi_{j}\right)^{2}$.

\section{B. Difficulties in the comparison with the theory}

When comparing the theory with the numerical data, we face two difficulties. First of all, the value of $\varphi_{j}$ depends on the numerical protocol and on its particular realization we used, and therefore cannot be directly compared with $\varphi_{G C P}$. Note that $\varphi_{G C P}$ should represent an upper bound for $\varphi_{j}$, whatever the protocol, but the value $\varphi_{G C P}=0.633353$ we report seems to contradict this statement. The reason is simply that our theoretical computations are based on the HNC equation of state for liquid hard spheres, which (as discussed above), underestimates the value of $\varphi_{G C P}$ : a better result is obtained using the Carnahan-Starling equation of state, which gives $\varphi_{G C P}=0.683$, consistently with the value of $\varphi_{j}$ found above [33]. To avoid confusion, here we stick to the use of the HNC equation of state; the theoretical calculations can be easily repeated for any other equation of state, and the scaling around $\varphi_{G C P}$ is unaffected by this choice. Once again we stress that the absolute values of density reported in this paper should always be taken with this caveat in mind. Since we are interested here mainly in the relative distance to jamming, which is $\varphi-\varphi_{j}$ for the numerical data, and $\varphi-\varphi_{G C P}$ for the theory, the absolute values of density are not crucial for our purposes. 
The second difficulty is the following. For hard spheres, the theory predicts that the reduced pressure diverges as $p_{\text {glass }}^{H S} \sim 3 \varphi_{G C P} /\left(\varphi_{G C P}-\varphi\right)$, while $y_{\text {glass }}^{H S}(\varphi) \sim$ $1.1 /\left(\varphi_{G C P}-\varphi\right)$ for $\varphi \rightarrow \varphi_{G C P}^{-}$, see Appendix E. As one can easily check, these scalings imply that the theory violates the exact thermodynamic relation $p_{\text {glass }}^{H S}=$ $1+4 \varphi y_{\text {glass }}^{H S}(\varphi)$, as already noticed in [33]. In particular, the divergence of $p_{\text {glass }}^{H S}$ is correct, while the coefficient of the divergence of $y_{\text {glass }}^{H S}(\varphi)$ is overestimated by a factor of $\sim 1.46562$. We will comment on the origin of this problem in Sec. VII. This error affects the prefactors in the scalings of $g_{\text {glass }}(r)$ and of $z$ around $\varphi_{G C P}$. To correct for this error, we rescaled the $\delta \varphi$ of the numerical data in such a way that the coefficient of the divergence of $y_{\text {glass }}^{H S}$ is the same as the theoretical one.

To summarize, when comparing numerical data with the theory, we consider $\delta \varphi=\varphi-\varphi_{G C P}$ for the theory, and $\delta \varphi=1.46562\left(\varphi-\varphi_{j}\right)$ for the numerical data such that the values of $y_{\text {glass }}^{H S}(\delta \varphi)$ for theory and simulations are exactly the same close to $\delta \varphi=0$. Note that this adjustment is based solely on the analysis of the hard sphere side of the jamming transition $(\delta \varphi<0)$, but we find that it allows to describe the full scaling also for positive $\delta \varphi$.

If the reader is uncomfortable with the above reasonings, another way of thinking is that we need to adjust two free parameters (the position of the transition and the amplitude of the rescaling of $\delta \varphi$ ) to obtain the best comparison of theory and numerical simulations. While the location is $\varphi_{j}$ was always adjusted in previous work [32, 38, 43, 44], we need to adjust also one prefactor as we seek to compare theoretical predictions to simulations in absolute values, and not only at the level of leading diverging contributions.

\section{Energy, pressure, average coordination}

We start our discussion by the simplest observables. In Fig. 12 we plot the average energy $U_{\text {glass }}(T, \varphi)$ and the inverse reduced pressure $1 / p_{\text {glass }}(T, \varphi)$ as functions of $\delta \varphi$ for several temperatures. The agreement between theory and numerical data, with the rescaling of $\delta \varphi$ discussed above, is nearly perfect. The scaling around jamming is clearly visible in the figures. For instance, $U_{\text {glass }}(T, \varphi)$ tends to a finite value for $\delta \varphi>0$, while for $\delta \varphi<0$ it goes to zero as a power law, since in this case the system becomes a hard sphere glass. Similarly, the reduced pressure is finite for $\delta \varphi<0$, while it diverges proportionally to $\beta$ for $\delta \varphi>0$, since in this case the pressure is finite at zero temperature. At finite temperature, the curves interpolate between the two regimes. Scaling functions similarly to the ones shown in Fig. 10 for $\mathrm{m}^{*}$ can easily be constructed.

In Fig. 13 we report the average coordination number $z(T, \varphi)$, as a function of $\delta \varphi$ for several temperatures. At $T=0$, the average coordination jumps from 0 to 6
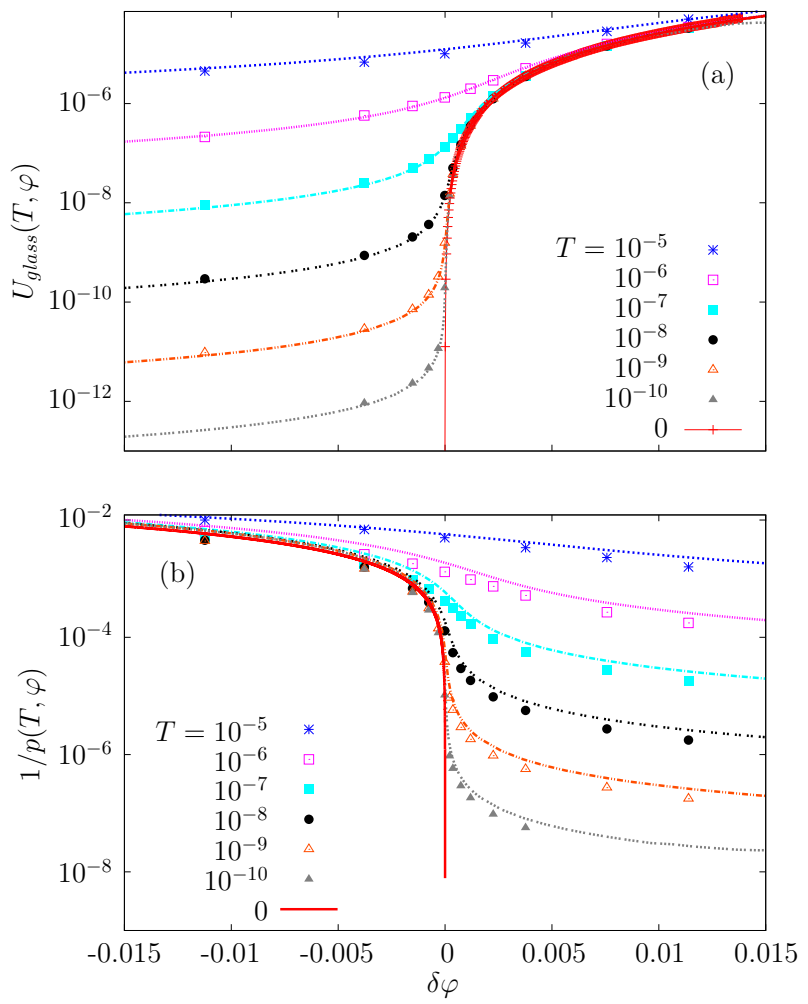

FIG. 12: (Color online) Energy $U_{\text {glass }}(T, \varphi)$ (top panel) and inverse reduced pressure $1 / p_{\text {glass }}(T, \varphi)$ (bottom panel) as functions of distance from jamming $\delta \varphi$, for several temperatures. We define $\delta \varphi=\varphi-\varphi_{G C P}$ for the theory, and $\delta \varphi=1.46562\left(\varphi-\varphi_{j}\right)$ for the numerical data.

(for numerical data) or to 6.13720 (for the theory). For $\delta \varphi>0$, the average coordination grows linearly in $\delta \varphi$ for the theory, while it grows as $\delta \varphi^{1 / 2}$ for the numerical data. Therefore, the theory fails to capture the correct evolution of this structural property at $\delta \varphi>0$ and $T=0$.

A more detailed description of what happens is obtained by looking to the data at finite $T$. When temperatures become too large, e.g. at $T=10^{-5}$, the theory eventually fails because of the low- $T$ approximation we made on the liquid theory in Eq. (21). For any fixed temperature $T \leq 10^{-6}$, we observe that the theory describes perfectly the numerical data for $\delta \varphi<0$ and also for positive $\delta \varphi$ up to some crossover value $\delta \varphi_{h}(T)$. Around $\delta \varphi_{h}(T)$ the theory starts deviating from the numerical data. The data in Fig. 13 suggest that $\delta \varphi_{h}(T)$ is an increasing function of $T$, i.e. that for higher temperatures the theory performs better, if the temperature is low enough that approximation (21) makes sense. Indeed, we expect that for $\delta \varphi_{h}(T) \rightarrow 0$ for $T \rightarrow 0$, since at $T=0$ the theory fails to capture the behavior of the contact number at $\delta \varphi>0$. We will come back to this point below. 


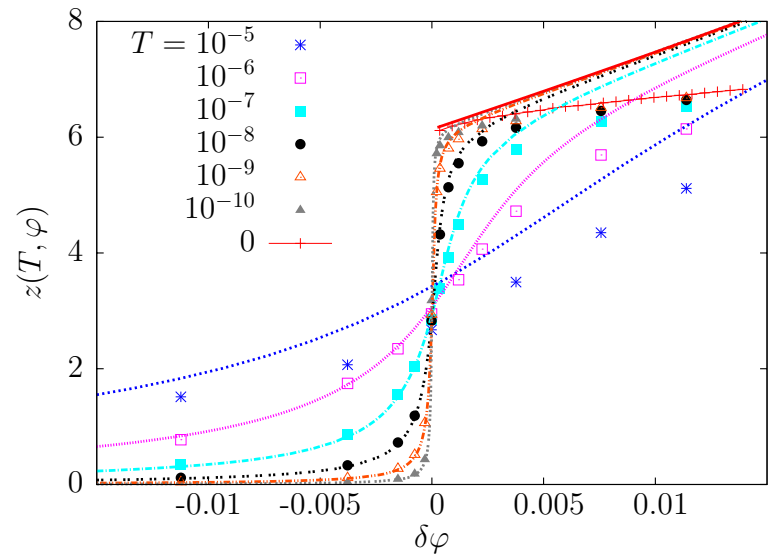

FIG. 13: (Color online) Average number of contacts $z$ as a function of distance from jamming $\delta \varphi$, for several temperatures. We define $\delta \varphi=\varphi-\varphi_{G C P}$ for the theory, and $\delta \varphi=1.46562\left(\varphi-\varphi_{j}\right)$ for the numerical data.

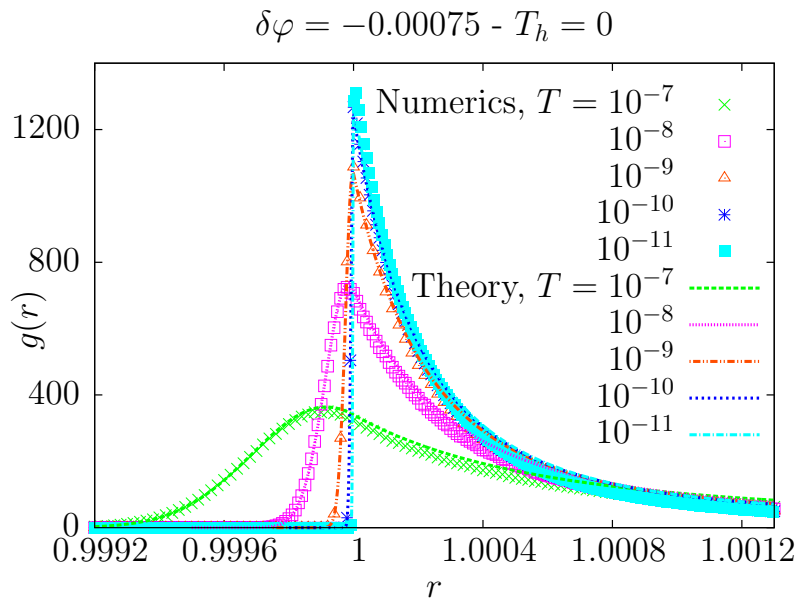

FIG. 14: (Color online) Pair correlation $g_{\text {glass }}(r)$ just below jamming predicted by theory (full lines) and measured in numerical simulations (symbols). Here $\delta \varphi=-0.00075$.

\section{Correlation function}

We now report data for the scaling of the contact peak of $g_{\text {glass }}(r)$ near jamming. We first consider a fixed $\delta \varphi<$ 0 , and change the temperature. In Fig. 14 we report data for $\delta \varphi=-0.00078$ for several temperatures. In this regime the theory works very well down to $T=0$; this is expected since we know that the theory works at $T=0$ for hard spheres [33].

Next, we consider a fixed $\delta \varphi>0$ and plot $g_{\text {glass }}(r)$ for several temperatures. This is done in Fig. 15 for four different values of $\delta \varphi$. Here, we observe that, like for the average coordination (Fig. 13), the theory works very well at moderately large $T$. However, on lowering $T$, at some point we observe that the numerical $g_{\text {glass }}(r)$ saturates to a limiting curve which has a maximum at $r<1$. By contrast, the theoretical curves slowly evolve towards the
$T=0$ theoretical limit, which is a half-Gaussian centered in $r=1$, with its $r>1$ part removed.

Therefore, below a certain temperature $T_{h}(\delta \varphi)$ (the inverse of the function $\delta \varphi_{h}(T)$ mentioned above), a deviation between theory and numerical data is observed close to the maximum of $g_{\text {glass }}(r)$. Clearly, the deviation between data and theory is a smooth crossover, so determining $T_{h}(\delta \varphi)$ is not easy. Here we choose the following procedure. The numerical data accumulate on a master curve as $T \rightarrow 0$. Therefore we define $T_{h}(\delta \varphi)$ as the value of temperature at which the theoretical curve best fits the $T \rightarrow 0$ numerical curve. As an example, in the upper left panel of Fig. 15, we see that the theoretical curve for $T=10^{-10}$ fits very well the numerical curves for both $T=10^{-10}, 10^{-11}$, while the $T=10^{-11}$ theoretical curve is quite different. We fix $T_{h}=10^{-10}$ for this value of $\delta \varphi$. Clearly the ambiguity on the precise numerical value of $T_{h}$ is rather large. Despite this, we are able to qualitatively confirm that $T_{h}(\delta \varphi)$ is an increasing function of $\delta \varphi$, as already suggested in the previous section. The function $T_{h}(\delta \varphi)$ determined from the pair correlation functions is reported in Fig. 16. It emerges continuously from zero above $\varphi_{G C P}$. A comparison with the temperature scale given by the glass temperature shows that it is orders of magnitude smaller than $T_{K}$. Thus it is only in the small regime of $T<T_{h}$ and $\varphi>\varphi_{G C P}$ that our the effective potential approach misses some important physics, as we discuss below in Sec. VIE.

Finally, in Fig. 17 we show that $g_{\text {glass }}(r)$, at $T=0$, follows the scaling relations predicted by the theory, Eqs. (54) and (60), for $|\delta \varphi| \rightarrow 0$, with different scaling functions on the two sides of the transition. While the scaling is perfect for $\delta \varphi<0$ [33], for $\delta \varphi>0$ there is a small deviation around the maximum as already discussed, which is however very small in the scaling regime for $\delta \varphi \rightarrow 0$.

\section{E. Discussion}

The main discrepancy between the theory and numerical data is in the region $\delta \varphi>0$ and very small temperature $T<T_{h}(\delta \varphi)$. Here, the average coordination scaling $z \propto \delta \varphi^{1 / 2}$ at $T=0$ is not obtained. Furthermore, the shape of the peak of $g_{\text {glass }}(r)$ is only partially captured by the theory. We tentatively attribute these discrepancies to the particular nature of the vibrational modes at $T=0$ and $\delta \varphi>0$. It has been shown that in this regime the low-frequency spectrum is dominated by spatially correlated vibrational modes. These modes cannot be described by our approach, in which vibrations are assumed to be Gaussian and only two-body correlations are taken into account. Therefore it is to be expected that the particular features of the jamming transition that are related to these correlated soft modes are not well reproduced by our theory.

On the hard sphere side, the soft modes induce a square-root divergence of the pair correlation function 

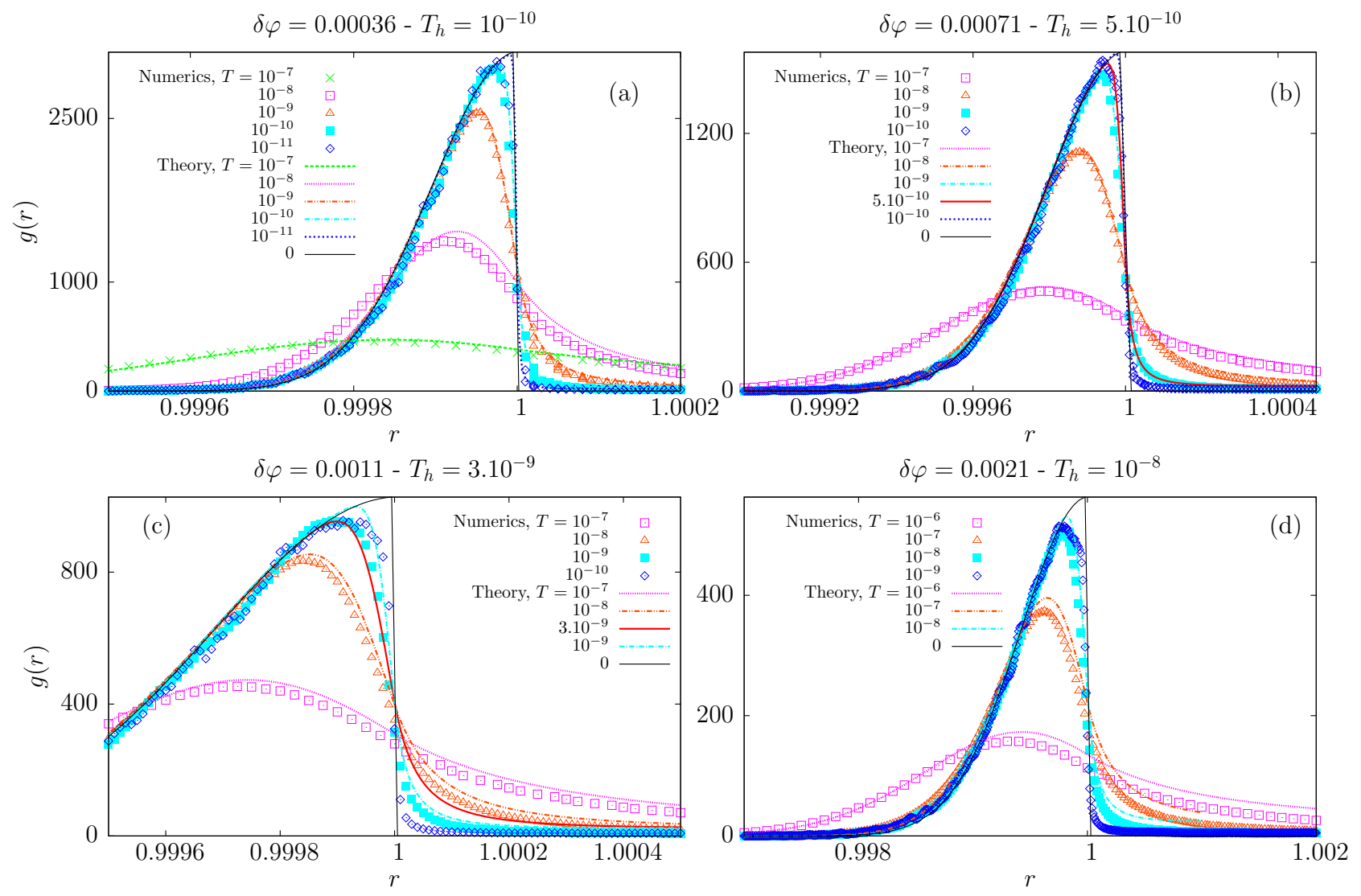

FIG. 15: (Color online) Pair correlations $g_{\text {glass }}(r)$ above jamming predicted by theory (full lines) and measured in numerical simulations (symbols). The agreement is very good for $T>T_{h}(\delta \varphi)$, the values of $T_{h}(\delta \varphi)$ are reported above the figures. Below $T_{h}(\varphi)$, the numerical curves do not evolve anymore, while the theoretical curves continue to evolve towards the $T=0$ theoretical limit.

$g_{\text {glass }}(r) \propto 1 / \sqrt{r-1}$. Yet, for $\delta \varphi \rightarrow 0^{-}$, this square root singularity is well separated from the contact peak [33]; therefore the shape of the scaling function for the contact peak is not affected by the soft modes and it is indeed correctly reproduced by the theory (see [33] and Fig. 17).

On the other side of the transition and for small $\delta \varphi>0$, it is commonly assumed that both the contact peak and the square root singularity are shifted by $\delta \varphi$. Under this assumption, the square root contribution should become $g_{\text {glass }}(r) \sim 1 / \sqrt{r-1+a \delta \varphi}$. Inserting this form of $g_{\text {glass }}(r)$ into Eq. (49) yields the result that $z=z_{0}+c \delta \varphi^{1 / 2}$, where $z_{0}$ is the contribution of the contact peak. While $z_{0}$ is clearly unaffected by the shift, the scaling of $z$ with $\delta \phi$ directly stems from the square root singularity of the pair correlation function. Therefore, the scaling of $z$ is dominated by the soft modes contribution, which might explain why it is not well captured by our theory.

On the other hand, inserting the same expression in the general formula for the internal energy, Eq. (26), one finds that $e_{G S} \sim \tilde{e} \delta \varphi^{2}+d \delta \varphi^{5 / 2}$, the first term being the contribution of the contact peak, the second being the one of the square root singularity. Therefore, for the energy the contribution of the anomalous modes is subdominant, and for this reason we observe that the theory reproduces well the numerical data in this case. The anomalous correction $\delta \varphi^{5 / 2}$ is of course not reproduced by the theory, that gives an expansion of $e_{G S}$ in integer powers of $\delta \varphi$, but these are subdominant terms.

We also suspect that the shape of $g_{\text {glass }}(r)$ near the peak is also influenced by a mixing of the contact peak with the square root singularity. While both contributions are well separated for $\varphi<\varphi_{j}$, this is not necessarily the case above jamming, which likely explains the small deviations observed in Figs. 15 and 17, but we could not find an empirical or scaling argument to disentangle both contributions.

Finally, it is interesting to remark that these deviations between theory and simulations are washed out by a finite temperature $T>T_{h}(\delta \varphi)$. We suspect that $T_{h}(\delta \varphi)$ represents a temperature below which the many-body direct interactions induced by the presence of many replicas become relevant (see the discussion in section II C). Above this crossover temperature, vibrations are probably much less correlated and our approximations become correct.

A possible interpretation is that the low-temperature 


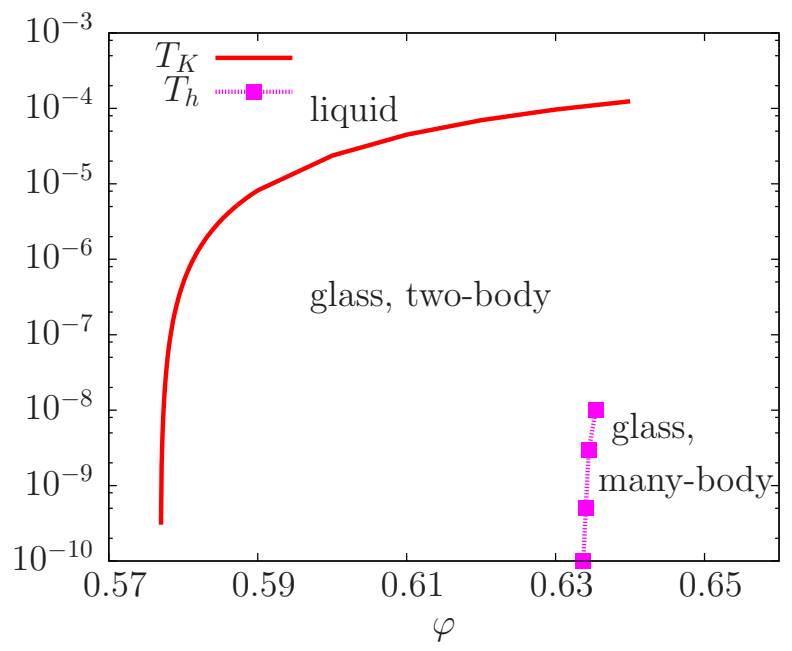

FIG. 16: (Color online) The crossover temperature $T_{h}(\varphi)$, as determined from Fig. 15, and compared with the Kauzmann temperature from Fig. 5.

correlated vibrations that are relevant below $T_{h}(\delta \varphi)$ are induced by the presence of strongly correlated soft vibrational modes at $T=0[14,32,38,56-60]$. Interestingly, the fact that $T_{h}(\delta \varphi) \rightarrow 0$ for $\delta \varphi \rightarrow 0^{+}$gives a hint that the correlations of these modes at $\delta \varphi=0$ are extremely fragile, such that an arbitrary small temperature is able to destroy them and restore the agreement between theory and numerical data. This observation is consistent with the numerical finding that around the jamming point, strong anharmonicity is present $[80,81]$ and the Hessian matrix of the potential does not capture the correct density of states of the vibrations [81].

\section{CONCLUSIONS}

In this final section we summarize our results and we discuss some perspectives for future work.

\section{A. Summary of results}

Understanding theoretically the physical properties of dense packings of soft repulsive particles is a fully nonequilibrium problem, because the jamming transition occurs in the absence of thermal fluctuations deep inside a glassy phase where phase space is dominated by the existence of a large number of metastable states. Athermal dynamical protocols sample packings with highly nonuniform weights [46, 62], while thermal protocols fall out of equilibrium at the glass transition [43].

Nevertheless, we showed that it can be successfully addressed using equilibrium statistical mechanics tools. We have developed a mean-field replica theory of the jamming transition of soft repulsive spheres which satisfactorily derives, from first principles, the existence and lo- cation of a jamming transition. This transition, that happens only at $T=0$, is exactly the same as the SAT/UNSAT transition of random constraint satisfaction problems $[48,49,52]$ : it is the point where the Parisi parameter $m^{*}$ that characterizes the 1RSB glass phase goes to zero, the reduced pressure of the hard sphere glass diverges, or equivalently its pressure and energy become finite. Within our approach, the jamming transition occurs deep into the glassy phase, and is thus a phenomenon which is physically distinct from the glass transition itself $[33,35]$. Finally, we have shown that the average coordination jumps from zero to $\sim 6$ at the transition, and we derived scaling functions that describe well the contact peak of the pair correlation function.

We want to stress here again that the absolute values of density reported in this paper are affected by the fact that we used the HNC equation of state for the liquid [33], and thus quantitative agreement with simulations cannot be expected. Still, we found that the scaling around jamming is almost insensitive to the particular equation of state that is chosen for the liquid. We also investigated the region of small $T$ around the jamming transition. We showed that the theory reproduces quite well the scaling of numerical data with $T$ and $\delta \varphi$, the distance from the jamming transition. On the hard spheres side of the transition $(\delta \varphi<0)$ the theory works well down to $T=0$. On the soft sphere side $(\delta \varphi>0)$ we have identified a temperature $T_{h}(\delta \varphi)$ below which, according to our interpretation, correlated vibrations $[56,58]$ that are neglected in our theory become relevant for some observables, and produce a series of interesting scalings that are not captured by our theory $[14,32,59]$. The most striking of these is the scaling of the number of contacts which our theory fails to reproduce. It is a major open problem to try and include in the theory a correct description of the divergent correlations in the vibrational modes $[56,58]$ that are relevant for $\delta \varphi>0$ and $T<T_{h}(\delta \varphi)$.

\section{B. Technical aspects}

There are several more technical aspects on which the theory could be improved, even in the region where it works quite well.

Our theory falls within the general framework of the replica method, using the molecular liquid formulation of $[30,31]$. However, to be able to describe the jamming transition, we had to develop a new approximation scheme, that is based on the effective potentials formulation of [33], and allows to write the molecular liquid as a simple liquid with effective potentials involving an arbitrary number of particles. Our approximation is based on the following steps:

1. As in [33], we neglect the three (and more)-body interactions, and just keep the two-body effective potential.

2. On top of this, we observe that the effective po- 

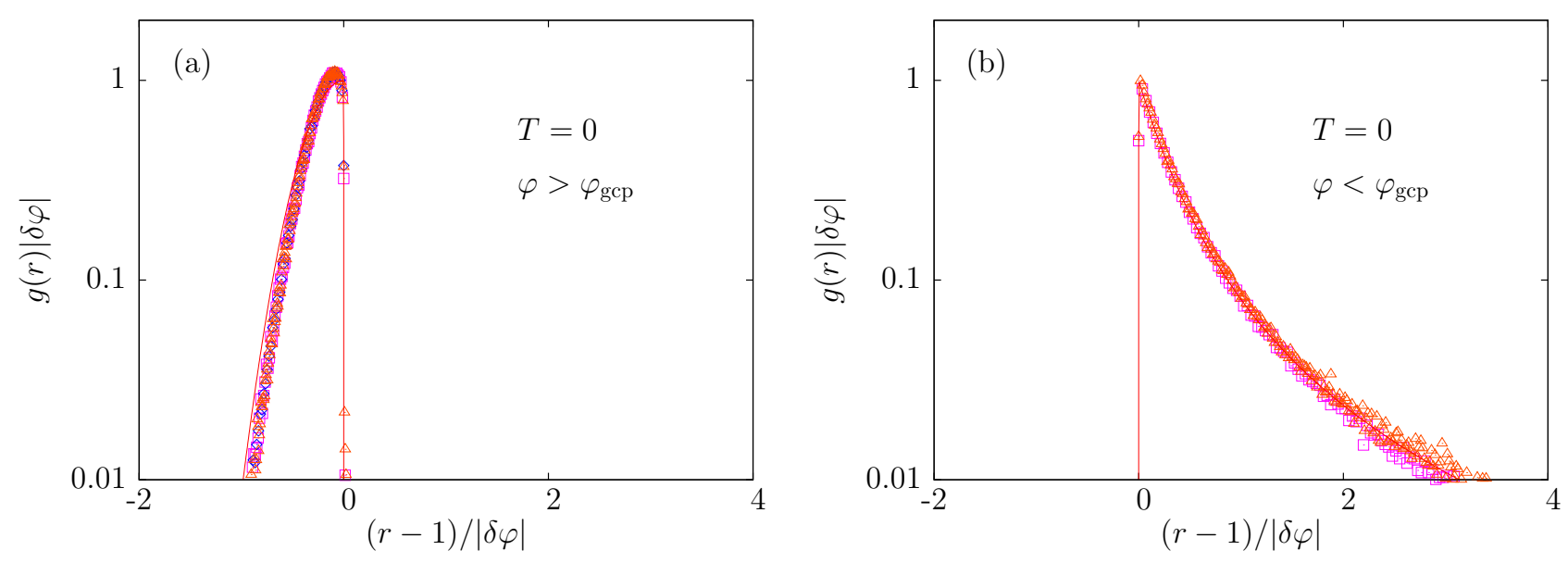

FIG. 17: (Color online) Scaling functions at $T=0$ above (left) and below (right) the jamming transition showing the convergence of the first peak near $r=1$ to a delta function with asymmetric scaling functions on both sides of the transition. To ease visualization, we show the evolution of $g(r) / g_{\max }$, where $g_{\max }$ can be read from Fig. 11 .

tential is given by the original potential (rescaled by a factor $m$ ) plus a small perturbation, therefore we use liquid perturbation theory to write the free energy of the replicated liquid as in Eq. (19).

3. Finally, we performed the low temperature approximation Eq. (21), in order to simplify the numerical calculations.

The last two approximations have been done mainly for practical reasons. However, we found that they are related to the first one. Indeed, if we get rid of approximation 3, we introduce an explicit dependence of $y_{\text {liq }}$ on temperature, hence on $\tau$ in the limit $T \rightarrow 0$ and $\delta \varphi>0$. It is easy to realize that this dependence affects the scaling close to jamming. An additional term $\sqrt{\tau}$ appears in the small $\tau$ expansion (see Appendix D 2), which implies that $\tau \propto \delta \varphi^{2}$ and $e \propto \delta \varphi^{3}$. An inspection of the small $\tau$ expansion reveals that if $y_{l i q}$ does not depend on $\tau$, then a delicate cancellation happens to eliminate the $\tau^{1 / 2}$ term and produce the correct scalings. The crucial observation is that the anomalous term contains the derivative of $y_{l i q}$ with respect to temperature, which is related to a three-body correlation function. Therefore we suspect that in a full treatment this term is cancelled by a term coming from the three body interaction potential. Therefore, getting rid of the third step of approximation might require taking into account three-body correlations as well, therefore getting rid of all steps at once.

This is of course an extremely interesting direction for future research, mainly because we suspect that getting rid of approximation 2 should allow to eliminate the violation of the exact thermodynamic relation $p_{\text {glass }}^{H S}=$ $1+4 \varphi y_{\text {glass }}^{H S}(\varphi)$ that happens in the theory and forces us to rescale $\delta \varphi$ in order to compare with numerical data. It is also possible that taking into account interactions involving several particles one could capture at least partially the long range correlations at jamming.
Finally, our theory neglects the existence of rattlers (particles with less than 4 contacts, which are therefore not mechanically blocked in jammed packings) since the cages are assumed to be identical for all particles [33]. Therefore the rattlers are approximated as jammed particles. This should not influence the shape of $g(r)$ close to the contact peak since typically rattlers are not in contact with other particles, so they do not contribute in this region, which was the main focus of this work. The theory can be in principle extended to include cage heterogeneity, and therefore describe rattlers. The computations will be more involved because a distribution of cage radii $P(A)$ should be included.

\section{Some general directions for future work}

Our approach is general enough that it can be systematically improved and generalized to various models (e.g. Hertzian potentials, or truncated Lennard-Jones potential). Moreover, recent work reported on a method to compute the shear modulus within the replica approach $[82,83]$. This could allow to add the external drive to the theory, and obtain a complete theoretical picture of the jamming transition in the three dimensional temperature, density, stress jamming phase diagram originally proposed by Liu and Nagel [13].

Finally, we believe that in the long term these studies could open a way towards a quantitative renormalization group treatment of structural glasses, following recent studies that formulated the renormalization group for lattice models $[67,68]$. If successful, this program could lead to a correct treatment of long-range correlations in the glass phase $[56,58]$, and in particular at the jamming point, and could also allow us to address the behavior of pair correlations over a broader range of distances, since pair correlation functions also exhibit singular behavior 
beyond the contact peak $[43,44]$, while the structure factor [84] and isothermal compressibility [85] also display intriguing anomalies at large scale which remain to be predicted theoretically.

\section{Acknowledgments}

We wish to thank D. Frenkel, A. Liu, G. Parisi, A. Sicilia, M. Wyart, and N. Xu for many useful discussions and comments on our paper and for sharing with us the results of their work. We thank F. van Wijland for giving us the opportunity to pursue our collaboration on this project. H. Jacquin PhD work is funded by a Fondation CFM-JP Aguilar grant. L. Berthier is partially funded by Région Languedoc-Roussillon.

\section{Appendix A: Complexity and the free-energy of basins}

The replica calculation presented in this paper is based on the assumption that phase space can be decomposed into a set of "pure states", which is the basis of Eq. (2). The precise definition of these states in statistical mechanics is however tricky and not very practical for numerical implementation. Although less rigorous, a more intuitive presentation in terms of the minima of the potential energy function, sometimes called "inherent structures" [86] or "basins", is very enlightening and allows an explicit connection with many numerical procedures encountered in the litterature to study the jamming transition $[38,69]$. For the sake of completeness, we include this discussion below. It is worth noting, however, that a partitioning of phase space in basins always depends on the details of the way these basins are defined. We expect that the particular definition we use below will be a good approximation of the equilibrium decomposition, at least for small enough temperatures.

\section{Definition of basins and their internal free energy}

We suppose that the phase space of all configurations of the spheres $\vec{R}$ can be unambiguously partitioned in basins. Each basin is labeled by a representative configuration, $\vec{R}_{0}$, see Fig. 18. For instance, one might think that a basin represents the set of configurations that end up in $\vec{R}_{0}$ after an energy minimization $\left(\vec{R}_{0}\right.$ is an energy minimum in this case). Or, for hard spheres, we can assume that a basin is the set of configurations that end up in $\vec{R}_{0}$ after a fast compression. One can, alternatively, minimize the energy first, then if the energy is zero (the configuration is not jammed), perform a fast compression, in such a way that $\vec{R}_{0}$ is always a jammed configuration. In any case, $\vec{R}_{0}$ will have minimal energy

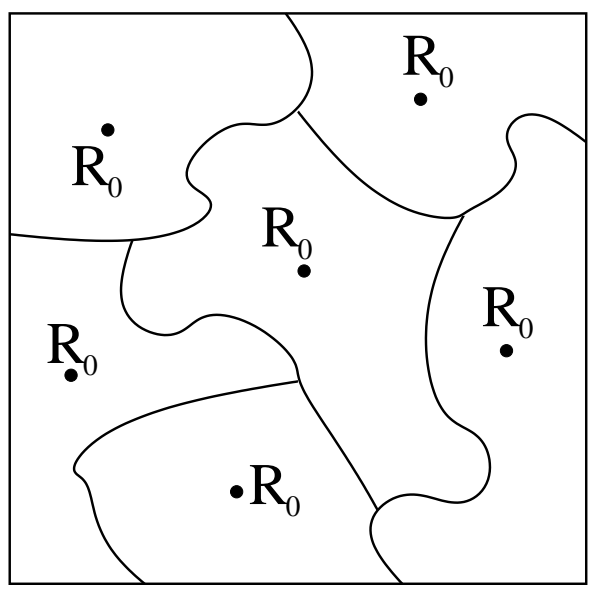

FIG. 18: A partitioning of phase space into basins, labeled by a representative configuration $\vec{R}_{0}$, which we assume to have minimal energy in the basin. Of course the shape of the basins is much more complex due to the high-dimensional nature of phase space. The above is just a very schematic representation.

in the basin at the end of the procedure. Note that the partition defined in this way is, a priori, dependent on the precise procedure used to perform the energy minimization or the compression. It is reasonable to expect that the ambiguity due to this is small and negligible in most cases.

The potential energy of the harmonic spheres is $V[\vec{R}]=$ $\sum_{\text {as }}$ i<j $\phi\left(\left|r_{i}-r_{j}\right|\right)$, and we define the free energy of a basin

$$
e^{-\beta N f\left[\vec{R}_{0}\right]}=\int d \vec{R} G\left(\vec{R}, \vec{R}_{0}\right) e^{-\beta V\left[\vec{R}_{0}\right]}
$$

where $G\left(\vec{R}, \vec{R}_{0}\right)=1$ if and only if configuration $\vec{R}$ belongs to basin $\vec{R}_{0}$. Note that for $\beta \rightarrow \infty$ we have

$$
e^{-\beta N f\left[\vec{R}_{0}\right]} \rightarrow \begin{cases}v_{b}\left(\vec{R}_{0}\right)=e^{N s\left[\vec{R}_{0}\right]} & \text { if } e\left[\vec{R}_{0}\right]=0 \\ e^{-\beta N e\left[\vec{R}_{0}\right]} & \text { if } e\left[\vec{R}_{0}\right]>0\end{cases}
$$

where $v_{b}$ is the volume of the configurations of the basin that are compatible with the hard sphere constraint (its logarithm being the internal entropy of the basin). Indeed, if the basin contains zero-energy configurations $\left(e\left[\vec{R}_{0}\right]=0\right)$, then at zero temperature its free energy is dominated by the number of those configurations. In the opposite case, if there are no hard sphere configurations $\left(e\left[\vec{R}_{0}\right]>0\right)$, it is very reasonable to assume that the minimum of the energy is unique and if we assume that $\vec{R}_{0}$ is the configuration of minimal energy inside the basin, then the weight is just dominated by its energy $e\left[\vec{R}_{0}\right]=V\left[\vec{R}_{0}\right] / N$. 


\section{The replica method and the complexity}

The replica method allows to compute the following object (for a given temperature $T$ and volume fraction $\varphi)$, see Eq. (9):

$$
Z_{m}=\sum_{\vec{R}_{0}} e^{-\beta m N f\left[\vec{R}_{0}\right]} .
$$

We can then compute a configurational entropy (or complexity) $\Sigma(m ; T, \varphi)$ according to Eq. (12):

$$
\begin{aligned}
\Sigma(m ; T, \varphi) & =-m^{2} \frac{d}{d m}\left[\frac{1}{m} \log Z_{m}\right] \\
& =-\sum_{\vec{R}_{0}} \frac{e^{-\beta m N f\left[\vec{R}_{0}\right]}}{Z_{m}} \log \left[\frac{e^{-\beta m N f\left[\vec{R}_{0}\right]}}{Z_{m}}\right] \\
& =-\sum_{\vec{R}_{0}} p_{\vec{R}_{0}} \log p_{\vec{R}_{0}}
\end{aligned}
$$

where

$$
p_{\vec{R}_{0}}=\frac{e^{-\beta m N f\left[\vec{R}_{0}\right]}}{Z_{m}} .
$$

Therefore, the complexity is the entropy of basins, weighted by the weight $p_{\vec{R}_{0}}$. The weight is constructed by assigning an "energy" $N f\left[\vec{R}_{0}\right]$ to each basin, and then constructing a Boltzmann weight at the effective temperature $\tau=T / m$.

\section{Special limits}

Equation (A4) allows to give simple interpretation to the different limits we considered in Sec. IV, as follows:

1. Consider $m=1$. Then the basins are weighted by their equilibrium free energy at temperature $T$. The associated complexity $\Sigma_{e q}(T, \varphi)=\Sigma(m=$ $1 ; T, \varphi)$ corresponds to the number of basins that dominate the equilibrium partition sum. In other words, if we take an equilibrium configuration at given $(T, \varphi)$, this configuration belongs, with probability 1 for $N \rightarrow \infty$, to one among $\exp \left[N \Sigma_{e q}\right]$ basins (all the other basins having a negligible probability at equilibrium). This equilibrium complexity is reported in Fig. 4.

2. Consider taking $\beta \rightarrow \infty$ first, then $m=1$. In this case we get, at low enough density where hard sphere configurations exist (omitting the normalization)

$$
p_{\vec{R}_{0}} \propto v_{b}\left[\vec{R}_{0}\right] .
$$

The basins are weighted by their hard sphere volume. Therefore $\Sigma_{e q}^{H S}(\varphi)=\Sigma(m=1 ; T=0, \varphi)$ is the equilibrium complexity of hard spheres: if one takes one hard sphere configuration at equilibrium at volume fraction $\varphi$, the latter will belong with probability 1 to one among $\exp \left[N \Sigma_{e q}^{H S}\right]$ basins. This quantity is reported in Fig. 16 of Ref. [33] and in the inset of Fig. 4.

3. Consider taking $m=0$. In this case, the basins are not weighted. Therefore, $\Sigma(m=0 ; T, \varphi)$ is just the logarithm of the total number of basins, of any energy and entropy.

4. Consider taking $\beta \rightarrow \infty$ first, again in the region of $\varphi$ where hard sphere configurations exist. Then, the weight becomes

$$
p_{\vec{R}_{0}} \propto v_{b}\left[\vec{R}_{0}\right]^{m}
$$

which means that basins are weighted by their volume of hard spheres configurations raised to the power $m$. In particular, those basins that do not contain any hard sphere configuration (those of positive energy) have zero weight and are not counted. Now, take $m \rightarrow 0$. The resulting weight is

$$
p_{\vec{R}_{0}} \propto \theta\left\{v_{b}\left[\vec{R}_{0}\right]>0\right\},
$$

in other words we give uniform weight to all basins that contain at least one hard sphere configuration. The corresponding complexity is

$$
\Sigma_{0}^{H S}(\varphi)=\lim _{m \rightarrow 0} \lim _{T \rightarrow 0} \Sigma(m ; T, \varphi)
$$

and it counts the total number of basins for hard spheres.

5. Finally, consider taking the limit $\beta \rightarrow \infty$, with the effective temperature $\tau=T / m$ held constant (meaning that $m \rightarrow 0$ at the same time). In this case, the weight becomes

$$
p_{\vec{R}_{0}} \propto e^{-N e\left[\vec{R}_{0}\right] / \tau} .
$$

Therefore, $\Sigma_{0}(\tau, \varphi)=\lim _{T \rightarrow 0} \Sigma(m=T / \tau ; T, \varphi)$ counts the basins weighted by their minimal energy at temperature $\tau$, irrespective of their entropy (in particular basins that contain hard sphere configurations have weight 1 in this case). Obviously, $\lim _{\tau \rightarrow 0} \Sigma_{0}(\tau, \varphi)=\Sigma_{0}^{H S}(\varphi)$ for $\varphi \leq \varphi_{G C P}$. Moreover, one can perform a Legendre transformation with respect to $\tau$ to eliminate $\tau$ in favor of its conjugate variable (the basins minimal energy) and therefore obtain $\Sigma_{0}(e, \varphi)$, which counts the basins of minimal energy $e$ at density $\varphi$. Again, $\lim _{e \rightarrow 0} \Sigma_{0}(e, \varphi)=\Sigma_{0}^{H S}(\varphi)$ for $\varphi \leq \varphi_{G C P}$, while for $\varphi>\varphi_{G C P}$, one finds that $\Sigma_{0}(e, \varphi)$ vanishes at a positive energy $e_{G S}(\varphi)$ which is the global minimum energy for that density and increases as $e_{G S}(\varphi) \propto\left(\varphi-\varphi_{G C P}\right)^{2}[54]$. The quantity $\Sigma_{0}(e, \varphi)$ is reported in Fig. 9. A similar object was recently measured numerically [69]. 


\section{Appendix B: Some calculations and simplications}

\section{The function $q$}

The function $q(A, T ; r)$ that appears in the effective potential Eq. (17) is given, using the definition and introducing bipolar coordinates, by:

$$
q(A, T ; r)=\int d^{3} r^{\prime} \gamma_{2 A}\left(\vec{r}^{\prime}\right) e^{-\beta \phi\left(\vec{r}-\vec{r}^{\prime}\right)}=\frac{1}{r \sqrt{4 \pi A}} \int_{0}^{\infty} d u u\left[e^{-\frac{(r-u)^{2}}{4 A}}-e^{-\frac{(r+u)^{2}}{4 A}}\right] e^{-\beta \phi(u)}
$$

The Gaussian integral can be explicitly computed and the result is

$$
\begin{aligned}
q(A, T ; r) & =\frac{1}{2}\left(2+\operatorname{Erf}\left[\frac{r-1}{2 \sqrt{A}}\right]-\operatorname{Erf}\left[\frac{r+1}{2 \sqrt{A}}\right]\right)+\frac{4 A^{3 / 2} \beta}{(1+4 A \beta) \sqrt{\pi} r}\left(e^{-\frac{(r-1)^{2}}{4 A}}-e^{-\frac{(r+1)^{2}}{4 A}}\right) \\
& +e^{-\frac{(r-1)^{2} \beta}{1+4 A \beta}} \frac{(r+4 A \beta)}{2 r(1+4 A \beta)^{3 / 2}}\left(\operatorname{Erf}\left[\frac{r+4 A \beta}{2 \sqrt{A(1+4 A \beta)}}\right]+\operatorname{Erf}\left[\frac{1-r}{2 \sqrt{A(1+4 A \beta)}}\right]\right) \\
& -e^{-\frac{(r+1)^{2} \beta}{1+4 A \beta}} \frac{(r-4 A \beta)}{2 r(1+4 A \beta)^{3 / 2}}\left(\operatorname{Erf}\left[\frac{r-4 A \beta}{2 \sqrt{A(1+4 A \beta)}}\right]-\operatorname{Erf}\left[\frac{1+r}{2 \sqrt{A(1+4 A \beta)}}\right]\right) .
\end{aligned}
$$

\section{Link with the Mézard-Parisi small cage expansion}

Here we show that our effective potential approximation reproduces the result of [31] in a small $A$ expansion at fixed $\beta$. Starting from Eq. (B1), we note that for small $A, \vec{r}^{\prime}$ is small, and we expand the potential $\phi\left(\vec{r}-\vec{r}^{\prime}\right)($ which is assumed to be differentiable) for small $\vec{r}^{\prime}$ :

$$
\begin{aligned}
q(A, T ; r) & =\int d^{3} r^{\prime} \gamma_{2 A}\left(\vec{r}^{\prime}\right) e^{-\beta \phi\left(\vec{r}-\vec{r}^{\prime}\right)} \\
& \sim e^{-\beta \phi(r)}\left[1-\beta A \Delta \phi(r)+\beta^{2} A(\nabla \phi(r))^{2}+O\left(A^{2}\right)\right]
\end{aligned}
$$

Plugging this in (23) and using an integration by parts, one easily obtains:

$$
G(m, A ; T)=3 \beta A(1-m) \int_{0}^{1} d r r^{2} e^{-\beta m \phi(r)} \Delta \phi(r)
$$

This result, together with Eq. (22), reproduces the result of [31], with the additional approximation we made for the correlation function of the liquid, Eq. (21). Note that without the latter approximation, we would not reproduce the result of [31] exactly; this would require an expansion around the center of mass of the molecule and not around replica 1 as we did in Eq. (15).

\section{Simplification of the replicated free entropy in the low-temperature approximation}

We show here the details of the simplification of the replicated free entropy in the low-temperature approximation, Eq. (15). Starting from Eq. (19) and using Eq. (15), we get

$$
\int d^{3} r g_{l i q}(T / m, \varphi ; r) Q(r) \sim y_{l i q}^{H S}(\varphi) \int d r e^{-\beta m \phi(r)} Q(r) \equiv y_{l i q}^{H S}(\varphi) \frac{4 \pi}{3} G(m, A ; T)
$$

where:

$$
G(m, A ; T)=\frac{3}{4 \pi} \int d^{3} r e^{-\beta m \phi(r)} Q(r)=3 \int_{0}^{\infty} d r r^{2}\left[e^{-\beta \phi_{e f f}(r)}-e^{-\beta m \phi(r)}\right] .
$$


Then we observe that, using Eqs. (14) and (15), we get

$$
\begin{aligned}
& \int d^{3} r e^{-\beta \phi_{e f f}(\vec{r})}=\frac{1}{V} \int d \vec{x}_{1} d \vec{y}_{1} e^{-\beta \phi_{e f f}\left(\vec{x}_{1}-\vec{y}_{1}\right)} \\
& =\frac{1}{V} \int d^{3} x_{1} \cdots d^{3} x_{m} d^{3} y_{1} \cdots d^{3} y_{m} \rho\left(\vec{x}_{1} \cdots \vec{x}_{m}\right) \rho\left(\vec{y}_{1} \cdots \vec{y}_{m}\right) \prod_{a=1}^{m} e^{-\beta \phi\left(\vec{x}_{a}-\vec{y}_{a}\right)}, \\
& \equiv \frac{1}{V} \int d^{3} X d^{3} Y q(A, T ; \vec{X}-\vec{Y})^{m}=\int d^{3} r q(A, T ; \vec{r})^{m},
\end{aligned}
$$

therefore we obtain

$$
G(m, A ; T)=3 \int_{0}^{\infty} d r r^{2}\left[q(A, T ; r)^{m}-e^{-\beta m \phi(r)}\right]
$$

which leads to the desired result, Eqs. (22) and (23).

\section{Energy and pressure of the glass}

Here we show how to compute the energy and pressure of the glass, which can be computed using the general thermodynamic relations $U=-d \mathcal{S} / d \beta$ and $p=\beta P / \rho=-\varphi d \mathcal{S} / d \varphi$, where $p$ is the so-called "reduced pressure" or "compressibility factor". From expression (13), together with $(22)$ and $(23)$, we have that $\mathcal{S}_{\text {glass }}(T, \varphi)=\mathcal{S}\left(m^{*}, A^{*} ; T, \varphi\right) / m^{*}$, where $m^{*}$ and $A^{*}$ are determined by optimization of $\mathcal{S}(m, A ; T, \varphi) / m$. Therefore, we do not need to take explicit derivatives with respect to $m$ and $A$.

We get for the energy:

$$
\begin{aligned}
U_{\text {glass }}(T, \varphi) & =-\frac{1}{m^{*}} \frac{\partial \mathcal{S}\left(m^{*}, A^{*} ; T, \varphi\right)}{\partial \beta} \\
& =-\frac{\partial \mathcal{S}_{l i q}\left(T / m^{*}, \varphi\right)}{\partial\left(\beta m^{*}\right)}-12 \varphi y_{\text {liq }}^{H S}(\varphi) \int_{0}^{\infty} d r r^{2}\left[q\left(A^{*}, T ; r\right)^{m^{*}-1} \frac{\partial q\left(A^{*}, T ; r\right)}{\partial \beta}+\phi(r) e^{\left.-\beta m^{*} \phi(r)\right]}\right. \\
& =U_{l i q}\left(T / m^{*}, \varphi\right)-12 \varphi y_{l i q}^{H S}(\varphi) \int_{0}^{\infty} d r r^{2} \phi(r) e^{-\beta m^{*} \phi(r)}-12 \varphi y_{l i q}^{H S}(\varphi) \int_{0}^{\infty} d r r^{2} q\left(A^{*}, T ; r\right)^{m^{*}-1} \frac{\partial q\left(A^{*}, T ; r\right)}{\partial \beta} \\
& =-12 \varphi y_{l i q}^{H S}(\varphi) \int_{0}^{\infty} d r r^{2} q\left(A^{*}, T ; r\right)^{m^{*}-1} \frac{\partial q\left(A^{*}, T ; r\right)}{\partial \beta},
\end{aligned}
$$

where we made use of Eq. (27) for the liquid energy. Now we make use of the definition of $q$, Eq. (B1), to get

$$
-\frac{\partial q(A, T ; r)}{\partial \beta}=\frac{1}{r \sqrt{4 \pi A}} \int_{0}^{\infty} d u u \phi(u)\left[e^{-\frac{(r-u)^{2}}{4 A}}-e^{-\frac{(r+u)^{2}}{4 A}}\right] e^{-\beta \phi(u)} .
$$

We plug this in the equation above, and we get (exchanging the names of $u$ and $r$ in the integral):

$$
\begin{aligned}
U_{\text {glass }}(T, \varphi) & =12 \varphi y_{\text {liq }}^{H S}(\varphi) \int_{0}^{\infty} d r r^{2} \phi(r) e^{-\beta \phi(r)} \frac{1}{r \sqrt{4 \pi A}} \int_{0}^{\infty} d u u\left[e^{-\frac{(r-u)^{2}}{4 A^{*}}}-e^{-\frac{(r+u)^{2}}{4 A^{*}}}\right] q\left(A^{*}, T ; u\right)^{m^{*}-1} \\
& =12 \varphi y_{\text {liq }}^{H S}(\varphi) \int_{0}^{\infty} d r r^{2} \phi(r) e^{-\beta \phi_{\text {eff }}(r)}
\end{aligned}
$$

where we used the definition (17). This is the desired result, Eq. (33).

For the pressure, we get the simple result

$$
\begin{aligned}
p_{\text {glass }}(T, \varphi) & =-\frac{\varphi}{m^{*}} \frac{\partial \mathcal{S}\left(m^{*}, A^{*} ; T, \varphi\right)}{\partial \varphi} \\
& =\frac{1}{m^{*}} p_{l i q}\left(T / m^{*}, \varphi\right)-\frac{4 \varphi}{m^{*}}\left[y_{l i q}^{H S}(\varphi)+\varphi \frac{d y_{l i q}^{H S}(\varphi)}{d \varphi}\right] G\left(m^{*}, A^{*}, T\right) .
\end{aligned}
$$




\section{Simplified expression of the number of contacts and of the energy}

Here we derive a simplified expression for the number of contacts. Using Eq. (15) we obtain

$$
\begin{aligned}
z(T, \varphi) & =24 \varphi y_{\text {liq }}^{H S}(\varphi) \int_{0}^{1} d r r^{2} e^{-\beta \phi_{\text {eff }}(r)} \\
& =24 \varphi y_{\text {liq }}^{H S}(\varphi) \int_{0}^{\infty} d u u^{2} q(A, T ; u)^{m-1} \frac{1}{u \sqrt{4 \pi A}} \int_{0}^{1} d r r\left[e^{-\frac{(r-u)^{2}}{4 A}}-e^{-\frac{(r+u)^{2}}{4 A}}\right] e^{-\beta(1-r)^{2}} \\
& =24 \varphi y_{\text {liq }}^{H S}(\varphi) \int_{0}^{\infty} d u u^{2} q(A, T ; u)^{m-1} \widehat{q}(A, T ; u)
\end{aligned}
$$

where we defined the function

$$
\widehat{q}(A, T ; u)=\frac{1}{u \sqrt{4 \pi A}} \int_{0}^{1} d r r\left[e^{-\frac{(r-u)^{2}}{4 A}}-e^{-\frac{(r+u)^{2}}{4 A}}\right] e^{-\beta(1-r)^{2}} .
$$

Note that $\widehat{q}(A, T ; u)$ is equal to the part of $q(A, T ; u)$ that vanishes in the zero temperature limit. This allows to compute it starting from Eq. (B2):

$$
\begin{aligned}
\widehat{q}(A, T ; r) & =q(A, T ; r)-\lim _{T \rightarrow 0} q(A, T ; r) \\
& =-\sqrt{\frac{A}{\pi}} \frac{1}{(1+4 A \beta) r}\left(e^{-\frac{(r-1)^{2}}{4 A}}-e^{-\frac{(r+1)^{2}}{4 A}}\right) \\
& +e^{-\frac{(r-1)^{2} \beta}{1+4 A \beta}} \frac{(r+4 A \beta)}{2 r(1+4 A \beta)^{3 / 2}}\left(\operatorname{Erf}\left[\frac{r+4 A \beta}{2 \sqrt{A(1+4 A \beta)}}\right]+\operatorname{Erf}\left[\frac{1-r}{2 \sqrt{A(1+4 A \beta)}}\right]\right) \\
& -e^{-\frac{(r+1)^{2} \beta}{1+4 A \beta}} \frac{(r-4 A \beta)}{2 r(1+4 A \beta)^{3 / 2}}\left(\operatorname{Erf}\left[\frac{r-4 A \beta}{2 \sqrt{A(1+4 A \beta)}}\right]-\operatorname{Erf}\left[\frac{1+r}{2 \sqrt{A(1+4 A \beta)}}\right]\right) .
\end{aligned}
$$

Similarly, for the energy of the glass we get

$$
\begin{aligned}
U_{\text {glass }}(T, \varphi) & =12 \varphi y_{\text {liq }}^{H S}(\varphi) \int_{0}^{1} d r r^{2}(1-r)^{2} e^{-\beta \phi_{\text {eff }}(r)} \\
& =12 \varphi y_{\text {liq }}^{H S}(\varphi) \int_{0}^{\infty} d u u^{2} q(A, T ; u)^{m-1} \frac{1}{u \sqrt{4 \pi A}} \int_{0}^{1} d r r(1-r)^{2}\left[e^{-\frac{(r-u)^{2}}{4 A}}-e^{-\frac{(r+u)^{2}}{4 A}}\right] e^{-\beta(1-r)^{2}} \\
& =12 \varphi y_{\text {liq }}^{H S}(\varphi) \int_{0}^{\infty} d u u^{2} q(A, T ; u)^{m-1}\left(-\frac{\partial \widehat{q}(A, T ; u)}{\partial \beta}\right)
\end{aligned}
$$

\section{Appendix C: The hard sphere limit}

\section{Free energy of hard spheres}

Here we discuss how Eq. (23) is simplified in the limit $T \rightarrow 0$, with fixed $m$ and $A$, that is the appropriate one below $\varphi_{G C P}$. Taking the limit $T \rightarrow 0$ at fixed $A$, Eq. (B2) gives back the results of Parisi and Zamponi [33, Eqs. (C4)]:

$$
q(A ; r)=\lim _{T \rightarrow 0} q(A, T ; r)=\frac{1}{2}\left(2+\operatorname{Erf}\left[\frac{r-1}{2 \sqrt{A}}\right]-\operatorname{Erf}\left[\frac{r+1}{2 \sqrt{A}}\right]\right)+\sqrt{\frac{A}{\pi}} \frac{1}{r}\left(e^{-\frac{(r-1)^{2}}{4 A}}-e^{-\frac{(r+1)^{2}}{4 A}}\right) .
$$

In this limit we obtain from Eq. (23) the following result:

$$
\begin{aligned}
& \mathcal{S}(m, A ; \varphi)=S_{h}(m, A)+\mathcal{S}_{l i q}^{H S}(\varphi)+4 \varphi y_{l i q}^{H S}(\varphi) G(m, A) \\
& G(m, A)=3 \int_{0}^{\infty} d r r^{2}\left[q(A ; r)^{m}-\theta(r-1)\right],
\end{aligned}
$$

These equations coincide with the ones reported in [33, Eq. (72)]. 


\section{Jamming limit from below}

The jamming limit from the hard sphere side $\varphi \rightarrow \varphi_{G C P}^{-}$is obtained by taking $m \rightarrow 0$ and $A=\alpha m$ [33]. In this limit, the function $q(A ; r)$ goes to 1 for $r>1$ and to 0 for $r<1$. Using the asymptotic expansions of the error function we get:

$$
q(A ; r) \sim \frac{1}{2}\left(1+\operatorname{Erf}\left[\frac{r-1}{2 \sqrt{A}}\right]\right)+\sqrt{\frac{A}{\pi}} \frac{1}{r} e^{-\frac{(r-1)^{2}}{4 A}} \sim \theta(r-1)+\sqrt{\frac{A}{\pi}} e^{-\frac{(r-1)^{2}}{4 A}}\left[\frac{1}{r}+\frac{1}{1-r} R\left(\frac{|1-r|}{\sqrt{A}}\right)\right]
$$

where the function $R(x)$ goes to 1 for $x \rightarrow \infty$ and is proportional to $x$ for $x \rightarrow 0$, in such a way that there is no singularity at $r=1$.

Based on this result, we can evaluate the integral that defines $G(m, A)$ in Eq. (C2). For $r>1$, we have $q(A ; r) \rightarrow 1$ and therefore

$$
\int_{1}^{\infty} d r r^{2}\left[q(m \alpha ; r)^{m}-1\right]=\int_{1}^{\infty} d r r^{2}\{m[q(m \alpha ; r)-1]+\cdots\}=S_{1} m^{2}+S_{2} m^{5 / 2}+\cdots
$$

For $r<1$, we have instead

$$
\int_{0}^{1} d r r^{2} q(m \alpha ; r)^{m} \sim\left(\sqrt{\frac{\alpha m}{\pi}}\right)^{m} \int_{0}^{1} d r r^{2} e^{-\frac{(r-1)^{2}}{4 \alpha}}\left[\frac{1}{r}+\frac{1}{1-r} R\left(\frac{1-r}{\sqrt{A}}\right)\right]^{m}
$$

The last term in square brackets in the integral gives rise to a regular expansion in powers of $m$ for small $m$. Collecting all these results together we get

$$
\begin{aligned}
& G(m, \alpha m) \sim m^{m / 2} G_{0}^{H S}(\alpha)\left(1+R_{1} m+R_{2} m^{2}+\cdots\right)+S_{1} m^{2}+S_{2} m^{5 / 2}+\cdots \\
& G_{0}^{H S}(\alpha)=3 \int_{0}^{1} r^{2} e^{-\frac{(1-r)^{2}}{4 \alpha}} d r
\end{aligned}
$$

Then, in the limit $m \rightarrow 0$, Eqs. (C2) reduce to:

$$
\begin{aligned}
& \mathcal{S}_{0}^{H S}(\alpha ; \varphi)=-\frac{3}{2}(\ln (2 \pi \alpha)+1)+\mathcal{S}_{\text {liq }}^{H S}(\varphi)+4 \varphi y_{\text {liq }}^{H S}(\varphi) G_{0}^{H S}(\alpha) \\
& G_{0}^{H S}(\alpha)=3 \int_{0}^{1} r^{2} e^{-\frac{(1-r)^{2}}{4 \alpha}} d r=3\left[\sqrt{\pi \alpha}(1+2 \alpha) \operatorname{Erf}\left(\frac{1}{2 \sqrt{\alpha}}\right)+2 \alpha e^{-\frac{1}{4 \alpha}}-4 \alpha\right] .
\end{aligned}
$$

Optimization over $\alpha$ yields an equation for $\alpha_{H S}^{*}(\varphi)$ :

$$
J_{0}^{H S}\left(\alpha_{H S}^{*}\right)=\frac{3}{8 \varphi y_{l i q}^{H S}(\varphi)}, \quad \text { with } \quad J_{0}^{H S}(\alpha)=\alpha \frac{d G_{0}^{H S}(\alpha)}{d \alpha} .
$$

Next we calculate the leading order in the small $m$ expansion around the previous result. We have

$$
\mathcal{S}(m, \alpha ; \varphi)=\mathcal{S}_{0}^{H S}(\alpha ; \varphi)+\frac{1}{2}\left[3+4 \varphi y_{\text {liq }}^{H S}(\varphi) G_{0}^{H S}(\alpha)\right] m \log m+O\left(m,(m \log m)^{2}, \cdots\right) .
$$

Therefore for the complexity we get at the leading order (recall that we do not need to add the explicit derivative with respect to $\alpha$, since $\alpha$ is defined by the condition that it vanishes):

$$
\Sigma(m, \alpha ; \varphi)=-m^{2} \frac{d}{d m}[\mathcal{S}(m, \alpha ; \varphi) / m]=\mathcal{S}_{0}^{H S}(\alpha ; \varphi)-\frac{m}{2}\left[3+4 \varphi y_{l i q}^{H S}(\varphi) G_{0}^{H S}(\alpha)\right]
$$

The expression above has to be computed in $\alpha=\alpha_{H S}^{*}(\varphi)$. Therefore, $\mathcal{S}_{0}^{H S}\left(\alpha^{*}(\varphi) ; \varphi\right)=\Sigma_{0}^{H S}(\varphi)$ is the complexity of hard-spheres at $m=0$ [33], which vanishes linearly at $\varphi=\varphi_{G C P}$. Since $m_{H S}^{*}(\varphi)$ is defined by $\Sigma\left(m, \alpha_{H S}^{*}(\varphi) ; \varphi\right)=0$, we get the leading term of $m_{H S}^{*}(\varphi)$ for $m \rightarrow 0$ or $\varphi \rightarrow \varphi_{G C P}^{-}$:

$$
m_{H S}^{*}(\varphi)=\frac{2 \Sigma_{0}^{H S}(\varphi)}{3+4 \varphi y_{l i q}^{H S}(\varphi) G_{0}^{H S}\left(\alpha_{H S}^{*}(\varphi)\right)}
$$




\section{Scaling of the effective potential on approaching jamming from below}

Here we discuss the emergence of the delta peak at $r=1^{+}$in the effective potential of hard spheres for $\varphi \rightarrow$ $\varphi_{G C P}^{-}[33]$, where $A=m \alpha$ and $m \rightarrow 0$ as discussed above. For small $A$, we have

$$
e^{-\beta \phi_{e f f}(r)}=\theta(r-1) \frac{1}{r \sqrt{4 \pi A}} \int_{0}^{\infty} d u u e^{-\frac{(r-u)^{2}}{4 A}} q(A ; u)^{m-1}
$$

with $q(A ; r)$ given in (C3). First we note that if $r-1 \gg \sqrt{A}$, then the integral is peaked at $u>1$, the function $q \rightarrow 1$ and the integral goes to 1 too. Therefore $e^{-\beta \phi_{e f f}(r)} \rightarrow 1$ for $r-1 \gg \sqrt{A}$.

We have then to study the region $r-1 \sim \sqrt{A}$. In this region one can easily show that the part of the integral at $u>1$ gives a finite contribution. If we want to study the divergent part, we can truncate the integral at $u=1$. We then introduce the scaling variable $\lambda=(r-1) / \sqrt{4 m A}=(r-1) /(m \sqrt{4 \alpha})>0$ and in the integral we change variable to $u=1-x \sqrt{4 A / m}=1-x \sqrt{4 \alpha}$. Using the first equation in (C3), and defining

$$
\Theta(s)=\frac{1}{2}[1+\operatorname{Erf}(s)]
$$

we get for the divergent part

$$
e^{-\beta \phi_{e f f}^{d i v}(\lambda)}=\frac{1}{\sqrt{\pi m}} \int_{0}^{1 / \sqrt{4 \alpha}} d x(1-x \sqrt{4 \alpha}) e^{-(\lambda \sqrt{m}+x / \sqrt{m})^{2}}\left[\Theta\left(-\frac{x}{\sqrt{m}}\right)+\sqrt{\frac{m}{\pi}} \frac{\sqrt{\alpha}}{1-x \sqrt{4 \alpha}} e^{-\frac{x^{2}}{m}}\right]^{m-1} .
$$

Making use of the asymptotic large $s$ expansion of the error function, we have $\Theta(-s) \sim e^{-s^{2}} /(2 \sqrt{\pi} s)$, and we get

$$
\begin{aligned}
e^{-\beta \phi_{e f f}^{d i v}(\lambda)} & =\frac{e^{-m \lambda^{2}}}{\sqrt{\pi m}} \int_{0}^{1 / \sqrt{4 \alpha}} d x(1-x \sqrt{4 \alpha}) e^{-2 \lambda x-x^{2}}\left[\sqrt{\frac{m}{\pi}} \frac{1}{2 x}+\sqrt{\frac{m}{\pi}} \frac{\sqrt{\alpha}}{1-x \sqrt{4 \alpha}}\right]^{m-1} \\
& \sim \frac{1}{m} \int_{0}^{1 / \sqrt{4 \alpha}} d x(1-x \sqrt{4 \alpha}) e^{-2 \lambda x-x^{2}}\left[\frac{1}{2 x}+\frac{\sqrt{\alpha}}{1-x \sqrt{4 \alpha}}\right]^{-1} \\
& =\frac{1}{m} \Delta_{\alpha}(\lambda),
\end{aligned}
$$

where we introduced the scaling function that describes the delta peak:

$$
\Delta_{\alpha}(\lambda)=2 \int_{0}^{1 / \sqrt{4 \alpha}} d x x(1-x \sqrt{4 \alpha})^{2} e^{-2 \lambda x-x^{2}}
$$

Note that at $\varphi_{G C P}$ we have $\alpha \sim 10^{-4}$; therefore an excellent approximation of the scaling function is obtained by setting $\alpha=0$ :

$$
\Delta_{0}(\lambda)=2 \int_{0}^{\infty} d x x e^{-2 \lambda x-x^{2}}=1-\sqrt{\pi} \lambda e^{\lambda^{2}}(1-\operatorname{Erf}(\lambda))
$$

that allows to recover the results of [33, Eq. (C40)].

\section{Appendix D: The zero temperature soft sphere limit}

\section{Free energy of soft spheres at $T=0$}

When $\varphi>\varphi_{G C P}$, the appropriate $T \rightarrow 0$ limit is taken with $m=T / \tau$ and $A=m \alpha$ for constants $\alpha$ and $\tau$. We start by looking at the asymptotics of $q(\alpha m, \tau m ; r)$ when $m$ goes to 0 . From the definition (B2) we find that:

$$
q(\alpha m, \tau m ; r) \underset{m \rightarrow 0}{\sim} \begin{cases}e^{-\frac{\phi(r)}{m(\tau+4 \alpha)} \frac{r+4 \alpha / \tau}{r(1+4 \alpha / \tau)^{3 / 2}}} & r<1 \\ 1 & r>1\end{cases}
$$


Therefore the part of the integrals in $G(m, A ; T)$ for $r>1$ vanishes in this limit. Plugging Eq. (D1) in Eq. (B8) we get:

$$
G(m, \alpha m ; \tau m) \underset{m \rightarrow 0}{\sim} 3 \int_{0}^{1} r^{2}\left[e^{-\frac{\phi(r) / \tau}{1+4 \alpha / \tau}}-e^{-\phi(r) / \tau}\right] d r
$$

which can be written, using the definition (C7), as:

$$
G(m, \alpha m ; \tau m) \underset{m \rightarrow 0}{\sim} G_{0}(\alpha, \tau)=G_{0}^{H S}(\alpha+\tau / 4)-G_{0}^{H S}(\tau / 4)
$$

The free entropy is given by Eq. (22). Replacing $T=\tau m$ and $A=\alpha m$ and taking the $m \rightarrow 0$ limit, we obtain:

$$
\mathcal{S}_{0}(\alpha, \tau ; \varphi)=-\frac{3}{2}(\ln (2 \pi \alpha)+1)+\mathcal{S}_{l i q}(\tau, \varphi)+4 \varphi y_{\text {liq }}^{H S}(\varphi) G_{0}(\alpha, \tau)
$$

As before, optimization over $\alpha$ yields an equation for $\alpha^{*}(\tau, \varphi)$ for each $\tau$ and $\varphi$ :

$$
\begin{aligned}
& J_{0}\left(\alpha^{*}(\tau, \varphi), \tau\right)=\frac{3}{8 \varphi y_{l i q}^{H S}(\varphi)}, \\
& J_{0}(\alpha, \tau)=\alpha \frac{d G_{0}}{d \alpha}(\alpha, \tau) .
\end{aligned}
$$

In this limit, the free energy is much simplified and the optimization over $\alpha$ and $\tau$ is easily carried out.

\section{Small $\tau$ expansion: jamming from above}

A very important observation is that, taking the $\tau \rightarrow 0$ limit, we recover the hard-sphere value of $\mathcal{S}_{0}^{H S}(\alpha ; \varphi)$ given in Eq. (C7). Indeed we expect that $\tau$ tends to zero when approaching $\varphi_{G C P}$ from above. Therefore, to expand around the jamming point, we must perform a small $\tau$ expansion of the free entropy (D4).

We start from (D4) and make use of the expressions (28) and (C7). We note that some remarkable cancellations happen, that eliminate a dangerous term in $\sqrt{\tau}$. Keeping only the lowest orders in the expression (D4) of the free entropy, we get:

$$
\begin{aligned}
& \mathcal{S}_{0}(\alpha, \tau ; \varphi)=\mathcal{S}_{0}^{H S}(\alpha ; \varphi)+\tau \mathcal{S}_{1}(\alpha, \varphi)+o(\tau), \\
& \mathcal{S}_{1}(\alpha, \varphi)=\varphi y_{\text {liq }}^{H S}(\varphi)\left[\frac{1}{\alpha} J_{0}^{H S}(\alpha)-6\right] .
\end{aligned}
$$

The optimal value of $\alpha$ is defined by :

$$
\frac{d \mathcal{S}_{0}^{H S}}{d \alpha}\left(\alpha^{*}(\tau, \varphi) ; \varphi\right)+\tau \frac{\partial \mathcal{S}_{1}}{\partial \alpha}\left(\alpha^{*}(\tau, \varphi), \varphi\right)=0,
$$

and it has the form

$$
\alpha^{*}(\tau, \varphi)=\alpha_{H S}^{*}(\varphi)+\tau b(\varphi)+o(\tau) .
$$

Recalling that $\alpha_{H S}^{*}$ maximizes $\mathcal{S}_{0}^{H S}(\alpha ; \varphi)$, therefore $\partial_{\alpha} \mathcal{S}_{0}^{H S}(\alpha ; \varphi)=0$ in $\alpha=\alpha_{H S}^{*}$, we see that the $\tau$-dependence of $\alpha^{*}$ does not contribute at lowest order. Thus:

$$
\mathcal{S}_{0}\left(\alpha^{*}(\tau, \varphi), \tau ; \varphi\right)=\mathcal{S}_{0}^{H S}\left(\alpha_{H S}^{*}(\varphi) ; \varphi\right)+\tau \mathcal{S}_{1}\left(\alpha_{H S}^{*}(\varphi), \varphi\right)+o(\tau)
$$

Remember that $\mathcal{S}_{0}^{H S}\left(\alpha^{*}(\varphi) ; \varphi\right)=\Sigma_{0}^{H S}(\varphi)$ is the complexity of hard-spheres at $m=0$ (see Appendix C). We define $\mathcal{S}_{1}(\varphi)=\mathcal{S}_{1}\left(\alpha_{H S}^{*}(\varphi), \varphi\right)$ and we obtain

$$
\mathcal{S}_{0}(\tau ; \varphi)=\Sigma_{0}^{H S}(\varphi)+\tau \mathcal{S}_{1}(\varphi)+o(\tau)
$$

Using Eq. (39), we obtain

$$
\begin{aligned}
& \Sigma_{0}(\tau, \varphi)=\Sigma_{0}^{H S}(\varphi)+2 \tau \mathcal{S}_{1}(\varphi) \\
& e(\tau, \varphi)=\tau^{2} \mathcal{S}_{1}(\varphi) .
\end{aligned}
$$

Finally, reconstructing this parametric equation defining $\Sigma_{0}$, we obtain :

$$
\Sigma_{0}(e, \varphi)=\Sigma_{0}^{H S}(\varphi)+2 \sqrt{e \mathcal{S}_{1}(\varphi)}
$$




\section{Effective potential in the zero temperature limit for soft spheres}

We start from the definition (17) of the effective potential and we take the limit $T \rightarrow 0$ with $m=T / \tau$ and $A=\alpha m$. A direct inspection of the different terms shows that, since $A$ is very small, one can discard a number of terms to obtain:

$$
\begin{aligned}
& e^{-\beta \phi_{e f f}(r)}=e^{-\beta \phi(r)} \frac{1}{r \sqrt{4 \pi A}} \int_{0}^{\infty} d u u e^{-\frac{(r-u)^{2}}{4 A}} q(A, T ; u)^{m-1}, \\
& q(A, T ; u) \sim \Theta\left(\frac{u-1}{\sqrt{4 A}}\right)+e^{-\frac{\beta(u-1)^{2}}{1+4 A \beta}} \frac{u+4 A \beta}{u(1+4 A \beta)^{3 / 2}} \Theta\left(\frac{1-u}{\sqrt{4 A(1+4 A \beta)}}\right),
\end{aligned}
$$

with $\Theta(s)$ defined in Eq. (C13). We should now make a separate analysis for $r>1$ and $r<1$. In both cases, the integral is dominated by $u \sim r$. Therefore, for $r>1$ also $u>1$ and in this case $q(A, T ; u) \sim 1$, so we get $e^{-\beta \phi_{e f f}(r)} \rightarrow 1$.

The more interesting case is for $r<1$. We have

$$
e^{-\beta \phi_{e f f}(r)}=e^{-\beta(1-r)^{2}} \int_{0}^{\infty} d u \frac{u e^{-\frac{(r-u)^{2}}{4 A}}}{r \sqrt{4 \pi A}}\left[\Theta\left(\frac{u-1}{\sqrt{4 A}}\right)+e^{-\frac{\beta(u-1)^{2}}{1+4 A \beta}} \frac{u+4 A \beta}{u(1+4 A \beta)^{3 / 2}} \Theta\left(\frac{1-u}{\sqrt{4 A(1+4 A \beta)}}\right)\right]^{m-1},
$$

For $r<1$, the integral over $u$ is dominated by the decay of the function away from $u=1$ (for $u<1$ ). We recall that $\Theta(s \rightarrow-\infty) \sim e^{-s^{2}}$, while $\Theta(s \rightarrow \infty) \rightarrow 1$. Therefore, inside the square brackets we can neglect the first term $\Theta\left(\frac{u-1}{\sqrt{4 A}}\right)$ whose decay is faster. We get, neglecting all the terms that are small for $m \rightarrow 0$

$$
\left.e^{-\beta \phi_{e f f}(r)}=e^{-\beta(1-r)^{2}} \frac{1}{r \sqrt{4 \pi \alpha m}} \int_{0}^{\infty} d u u e^{\frac{1}{m}\left\{-\frac{(r-u)^{2}}{4 \alpha}+\frac{(u-1)^{2}}{\tau+4 \alpha}\right.}\right\} e^{-\frac{(u-1)^{2}}{\tau+4 \alpha}} \frac{u(1+4 \alpha / \tau)^{3 / 2}}{u+4 \alpha / \tau} .
$$

The term in the exponential proportional to $1 / m$ dominates. Rearranging terms we obtain:

$$
\begin{aligned}
e^{-\beta \phi_{e f f}(r)} & =e^{-\frac{(1-r)^{2}}{m \tau}} e^{\frac{(r-1)^{2}}{m \tau}} \frac{1}{r} \int_{0}^{\infty} d u u \frac{e^{-\frac{[u+4 \alpha / \tau-r(1+4 \alpha / \tau)]^{2}}{4 \alpha m(1+4 \alpha / \tau}}}{\sqrt{4 \pi \alpha m(1+4 \alpha / \tau)}} e^{-\frac{(u-1)^{2}}{\tau+4 \alpha}} \frac{u(1+4 \alpha / \tau)^{2}}{u+4 \alpha / \tau} \\
& =\frac{1}{r} \int_{0}^{\infty} d u u \delta[u-1-(r-1)(1+4 \alpha / \tau)] e^{-\frac{(u-1)^{2}}{\tau+4 \alpha}} \frac{u(1+4 \alpha / \tau)^{2}}{u+4 \alpha / \tau} \\
& =\theta\left(r-\frac{4 \alpha}{\tau+4 \alpha}\right) \frac{(1+4 \alpha / \tau)[1+(r-1)(1+4 \alpha / \tau)]^{2}}{r^{2}} e^{-\frac{\tau+4 \alpha}{\tau^{2}}(r-1)^{2}}
\end{aligned}
$$

since the delta function forces $u=1+(r-1)(1+4 \alpha / \tau)$, but the integral vanishes unless this value of $u$ is positive. Adding the contribution for $r>1$, we get the final result:

$$
e^{-\beta \phi_{e f f}(r)}=\theta(r-1)+\theta(1-r) \theta\left(r-\frac{4 \alpha}{\tau+4 \alpha}\right) \frac{(1+4 \alpha / \tau)[1+(r-1)(1+4 \alpha / \tau)]^{2}}{r^{2}} e^{-\frac{\tau+4 \alpha}{\tau^{2}}(r-1)^{2}} .
$$

Note that the above expression is discontinuous in $r=1$, while at any finite $T$ the effective potential is continuous. We can derive the scaling function that describes the emergence of the discontinuity for $T \rightarrow 0$ as follows. We start from Eq. (D15) and we perform a change of variable $u=1+x \sqrt{4 A}$ in the integral, and define $\lambda=(r-1) / \sqrt{4 A}$. Then we get in the zero temperature limit

$$
\begin{aligned}
& e^{-\beta \phi_{e f f}(r)}=e^{-\frac{4 \alpha}{\tau} \lambda^{2} \theta(-\lambda)} \frac{1}{\sqrt{\pi}} \int_{-\infty}^{\infty} d x e^{-(\lambda-x)^{2}} \frac{1}{q_{0}(\alpha, \tau ; x)} \\
& q_{0}(\alpha, \tau ; x)=\Theta(x)+\frac{1}{\sqrt{1+4 \alpha / \tau}} \Theta\left(-\frac{x}{\sqrt{1+4 \alpha / \tau}}\right) e^{-\frac{4 \alpha}{\tau+4 \alpha} x^{2}}
\end{aligned}
$$

which provides the scaling function in the region where $\lambda$ is of order one, and $r-1 \sim \sqrt{A}$. It is quite easy to check that the function above tends to 1 for $\lambda \rightarrow \infty$, while it tends to $1+4 \alpha / \tau$ for $\lambda \rightarrow-\infty$, therefore interpolating between the two regimes $r>1$ and $r<1$. 


\section{Appendix E: Numerical calculations}

In this Appendix we collect all the numerical results of the various calculations presented in the paper.

\section{Hard spheres}

We start from the determination of $\varphi_{G C P}$, which is done in the most precise way by looking at the point where $\Sigma_{0}^{H S}(\varphi)=0$, see Appendix C and D 2. We get

$$
\begin{aligned}
& \varphi_{G C P}=0.633353, \\
& \alpha_{0}^{H S}\left(\varphi_{G C P}\right)=9.72187 \cdot 10^{-5},
\end{aligned}
$$

with $y_{l i q}^{H S}\left(\varphi_{G C P}\right)=23.6238$, and from Eq. (C11), using $G_{0}^{H S}\left(\alpha_{H S}^{*}\left(\varphi_{G C P}\right)\right)=0.0170908$, we get

$$
m_{H S}^{*}(\varphi)=\widetilde{\mu}\left(\varphi_{G C P}-\varphi\right)=20.7487\left(\varphi_{G C P}-\varphi\right) .
$$

From this and from Eq. (34) we can get the scaling of the reduced pressure for $\varphi \rightarrow \varphi_{G C P}^{-}$:

$$
\begin{aligned}
p_{\text {glass }} & \sim \frac{1}{\widetilde{\mu}\left(\varphi_{G C P}-\varphi\right)}\left[p_{l i q}^{H S}\left(\varphi_{G C P}\right)-4 \varphi_{G C P}\left(y_{\text {liq }}^{H S}\left(\varphi_{G C P}\right)+\varphi_{G C P} \frac{d y_{\text {liq }}^{H S}}{d \varphi}\left(\varphi_{G C P}\right)\right) G_{0}^{H S}\left(\alpha_{H S}^{*}\left(\varphi_{G C P}\right)\right)\right] \\
& =\frac{3.03430 \varphi_{G C P}}{\varphi_{G C P}-\varphi} .
\end{aligned}
$$

The prefactor 3.03430 is extremely close to the space dimension $d=3$, as predicted by free volume theory; the value $d$ is obtained in the small $A$ expansion [33].

\section{Soft spheres at zero temperature}

Close to $\varphi_{G C P}$ we have $\Sigma_{0}^{H S}(\varphi)=62.9577\left(\varphi_{G C P}-\varphi\right)$ and $\mathcal{S}_{1}\left(\varphi_{G C P}\right)=3767.51$, hence we immediately deduce from Eqs. (D11) that:

$$
\begin{aligned}
& \tau^{*}(\varphi)=-\Sigma_{0}^{H S}(\varphi) /\left(2 \mathcal{S}_{1}(\varphi)\right)=0.00835535\left(\varphi-\varphi_{G C P}\right), \\
& e_{G S}(\varphi)=\left(\Sigma_{0}^{H S}(\varphi)\right)^{2} /\left(4 \mathcal{S}_{1}(\varphi)\right)=0.263017\left(\varphi-\varphi_{G C P}\right)^{2}
\end{aligned}
$$

The pressure is obtained from Eq. (34):

$$
P_{\text {glass }}(T=0, \varphi)=\lim _{T \rightarrow 0}\left[\rho T p_{\text {glass }}(T, \varphi)\right]=\frac{6 \varphi}{\pi}\left[p_{\text {liq }}^{H S}(\varphi)-4 \varphi\left(y_{\text {liq }}^{H S}(\varphi)+\varphi \frac{d y_{\text {liq }}^{H S}}{d \varphi}(\varphi)\right) G_{0}\left(\alpha^{*}(\varphi), \tau^{*}(\varphi)\right)\right] \tau^{*}(\varphi),
$$

and expanding around $\varphi_{G C P}$ we get

$$
\begin{aligned}
P_{\text {glass }}(T=0, \varphi) & =\frac{6 \varphi_{G C P}}{\pi}\left[p_{l i q}^{H S}\left(\varphi_{G C P}\right)-4 \varphi_{G C P}\left(y_{\text {liq }}^{H S}\left(\varphi_{G C P}\right)+\varphi_{G C P} \frac{d y_{\text {liq }}^{H S}}{d \varphi}\left(\varphi_{G C P}\right)\right) G_{0}^{H S}\left(\alpha_{H S}^{*}\left(\varphi_{G C P}\right)\right)\right] \tau^{*}(\varphi) \\
& =0.403001\left(\varphi-\varphi_{G C P}\right) .
\end{aligned}
$$

Clearly the same scaling could have been obtained from the exact relation $P_{\text {glass }}(T=0, \varphi)=\frac{6 \varphi^{2}}{\pi} \frac{d e_{G S}}{d \varphi}$.

\section{Correlation functions}

From these values we can deduce the scaling of the peak of $g_{\text {glass }}(r)$ for $\varphi \rightarrow \varphi_{G C P}^{+}$:

$$
g_{\max }\left(\varphi \rightarrow \varphi_{G C P}^{+}\right)=y_{l i q}^{H S}(\varphi)\left(1+\frac{4 \alpha^{*}(\varphi)}{\tau^{*}(\varphi)}\right)=\frac{1.09949}{\varphi-\varphi_{G C P}},
$$


and for $\varphi \rightarrow \varphi_{G C P}^{-}$:

$$
g_{\max }\left(\varphi \rightarrow \varphi_{G C P}^{-}\right)=y_{l i q}^{H S}(\varphi) \frac{1}{m_{H S}^{*}(\varphi)} \Delta_{\alpha^{*}}(0)=\frac{1.09922}{\varphi_{G C P}-\varphi}
$$

Note that even though the prefactors are almost identical numerically, we were not able to prove that they actually coincide.

[1] J. D. Bernal, Nature 183, 141 (1959).

[2] J. D. Bernal and J. Mason, Nature 188, 910 (1960).

[3] H. M. Jaeger, S. R. Nagel, and R. P. Behringer, Rev. Mod. Phys. 68, 1259 (1996).

[4] R. G. Larson, The Structure and Rheology of Complex Fluids (Oxford University Press, 1999).

[5] J. P. Hansen and I. R. McDonald, Theory of simple liquids (Academic Press, London, 1986).

[6] T. Aste and D. Weaire, The pursuit of perfect packing (Taylor and Francis, 2000).

[7] S. Torquato and F. H. Stillinger, Rev. Mod. Phys. 82, 2633 (2010).

[8] S. F. Edwards and R. B. S. Oakeshott, Physica A 157, 1080 (1989).

[9] S. F. Edwards, Physica A 249, 226 (1998).

[10] C. Song, P. Wang, and H. A. Makse, Nature 453, 629 (2008).

[11] S. Henkes and B. Chakraborty, Phys. Rev. E 79, 061301 (2009).

[12] L. Berthier and G. Biroli, Rev. Mod. Phys. 83, 587 (2011).

[13] A. J. Liu and S. R. Nagel, Nature 396, 21 (1998).

[14] A. J. Liu, S. R. Nagel, W. van Saarloos, and M. Wyart, in Dynamical Heterogeneities in Glasses, Colloids, and Granular Media, edited by L. Berthier, G. Biroli, J.-P. Bouchaud, L. Cipeletti, and W. van Saarloos (Oxford University Press, 2011).

[15] H. Jacquin and L. Berthier, Soft Matter 6, 2970 (2010).

[16] L. Berthier, H. Jacquin, and F. Zamponi, J. Stat. Mech. p. P01004 (2010).

[17] J. P. Stoessel and P. G. Wolynes, J. Chem. Phys. 80, 4502 (1984).

[18] Y. Singh, J. P. Stoessel, and P. G. Wolynes, Phys. Rev. Lett. 54, 1059 (1985).

[19] T. R. Kirkpatrick and P. G. Wolynes, Phys. Rev. A 35, 3072 (1987).

[20] T. R. Kirkpatrick and D. Thirumalai, Phys. Rev. Lett. 58, 2091 (1987).

[21] T. R. Kirkpatrick, D. Thirumalai, and P. G. Wolynes, Phys. Rev. A 40, 1045 (1989).

[22] M. Mézard, G. Parisi, and M. A. Virasoro, Spin glass theory and beyond (World Scientific, Singapore, 1987).

[23] T. Castellani and A. Cavagna, J. Stat. Mech. p. P05012 (2005).

[24] V. Lubchenko and P. G. Wolynes, Ann. Rev. Phys. Chem. 58, 235 (2007).

[25] A. Cavagna, Phys. Rep. 476, 51 (2009).

[26] G. Biroli and J.-P. Bouchaud, arXiv:0912.2542 (2009).

[27] R. Monasson, Phys. Rev. Lett. 75, 2847 (1995).

[28] M. Mézard and G. Parisi, J. Phys. A 29, 6515 (1996).
[29] M. Cardenas, S. Franz, and G. Parisi, J. Phys. A 31, 163 (1998).

[30] M. Mézard and G. Parisi, J. Chem. Phys. 111, 1076 (1999).

[31] M. Mézard and G. Parisi, Phys. Rev. Lett. 82, 747 (1999).

[32] M. van Hecke, J. Phys.: Condens. Matter 22, 033101 (2010).

[33] G. Parisi and F. Zamponi, Rev. Mod. Phys. 82, 789 (2010).

[34] R. Mari and J. Kurchan, arXiv:1104.3420 (2011).

[35] R. Mari, F. Krzakala, and J. Kurchan, Phys. Rev. Lett. 103, 025701 (2009).

[36] M. Mézard, G. Parisi, M. Tarzia, and F. Zamponi, J. Stat. Mech. p. P03002 (2011).

[37] D. J. Durian, Phys. Rev. Lett. 75, 4780 (1995).

[38] C. S. O'Hern, S. A. Langer, A. J. Liu, and S. R. Nagel, Phys. Rev. Lett. 88, 075507 (2002).

[39] L. Berthier and T. A. Witten, Europhys. Lett. 86, 10001 (2009).

[40] L. Berthier and T. A. Witten, Phys. Rev. E 80, 021502 (2009).

[41] Z. Zhang, N. Xu, D. T. N. Chen, P. Yunker, A. M. Alsayed, K. B. Aptowicz, P. Habdas, A. J. Liu, S. R. Nagel, and A. G. Yodh, Nature 459, 230 (2009).

[42] A. Fernandez-Nieves, H. Wyss, J. Mattsson, and D. A. Weitz, eds., Microgel suspensions (Wiley-VCH, 2011).

[43] A. Donev, S. Torquato, and F. H. Stillinger, Phys. Rev. E 71, 011105 (2005).

[44] L. E. Silbert, A. J. Liu, and S. R. Nagel, Phys. Rev. E 73, 041304 (2006).

[45] C. Heussinger and J.-L. Barrat, Phys. Rev. Lett. 102, 218303 (2009).

[46] G.-J. Gao, J. Blawzdziewicz, and C. S. O'Hern, Phys. Rev. E 74, 061304 (2006).

[47] F. Krzakala and J. Kurchan, Phys. Rev. E 76, 021122 (2007).

[48] R. Monasson, in Complex Systems, edited by J.-P. Bouchaud, M. Mézard, and J. Dalibard (Elsevier, 2007).

[49] M. Mézard and A. Montanari, Information, Physics, and Computation (Oxford University Press, 2009).

[50] M. Mézard and G. Parisi, J. Stat. Phys. 111, 1 (2003).

[51] F. Krzakala, A. Montanari, F. Ricci-Tersenghi, G. Semerjian, and L. Zdeborová, Proc. Nat. Acad. Sci. 104, 10318 (2007).

[52] F. Krzakala and L. Zdeborová, EPL 81, 57005 (2008).

[53] F. Ricci-Tersenghi, Science 330, 1639 (2010).

[54] H. Jacquin, L. Berthier, and F. Zamponi, Phys. Rev. Lett. 106, 135702 (2011).

[55] M. Clusel, E. I. Corwin, A. O. N. Siemens, and J. Brujic, 
Nature 460, 611 (2009).

[56] M. Wyart, S. Nagel, and T. Witten, Europhysics Letters 72, 486 (2005).

[57] M. Wyart, L. E. Silbert, S. R. Nagel, and T. A. Witten, Phys. Rev. E 72, 051306 (2005).

[58] C. Brito and M. Wyart, Europhysics Letters (EPL) 76, 149 (2006).

[59] M. Wyart, Annales de Physique 30, 1 (2005), arXiv: cond-mat/0512155.

[60] C. S. O'Hern, L. E. Silbert, A. J. Liu, and S. R. Nagel, Phys. Rev. E 68, 011306 (2003).

[61] V. Vitelli, N. Xu, M. Wyart, A. J. Liu, and S. R. Nagel, Phys. Rev. E 81, 021301 (2010).

[62] G.-J. Gao, J. Blawzdziewicz, C. S. O'Hern, and M. Shattuck, Phys. Rev. E 80, 061304 (2009).

[63] Y. Jin and H. Makse, Physica A: Statistical Mechanics and its Applications 389 (2010).

[64] C. F. Schreck, C. S. O'Hern, and L. E. Silbert, Phys. Rev. E 84, 011305 (2011).

[65] Y. Jiao, F. Stillinger, and S. Torquato, Journal of Applied Physics 109, 013508 (2011).

[66] W. Kauzmann, Chem. Rev. 43, 219 (1948).

[67] M. Castellana, A. Decelle, S. Franz, M. Mézard, and G. Parisi, Phys. Rev. Lett. 104, 127206 (2010).

[68] C. Cammarota, G. Biroli, M. Tarzia, and G. Tarjus, Phys. Rev. Lett. 106, 115705 (2011).

[69] N. Xu, D. Frenkel, and A. J. Liu, Phys. Rev. Lett. 106, 245502 (2011).

[70] L. Foini, G. Semerjian, and F. Zamponi, Phys. Rev. B 83, 094513 (2011).
[71] L. Berthier, E. Flenner, H. Jacquin, and G. Szamel, Phys. Rev. E 81, 031505 (2010).

[72] J. H. Gibbs, J. Chem. Phys. 25, 185 (1956).

[73] J. H. Gibbs and E. A. Di Marzio, J. Chem. Phys. 28, 373 (1958).

[74] G. Adam and J. H. Gibbs, J. Chem. Phys. 43, 139 (1965).

[75] L.-M. Martinez and C. A. Angell, Nature 410, 663 (2001).

[76] Z. W. Salsburg and W. W. Wood, J. Chem. Phys. 37, 798 (1962)

[77] P. Olsson and S. Teitel, Phys. Rev. Lett. 99, 178001 (2007).

[78] X. Cheng, Phys. Rev. E 81, 031301 (2010).

[79] P. Chaudhuri, L. Berthier, and S. Sastry, Phys. Rev. Lett. 104, 165701 (2010).

[80] N. Xu, V. Vitelli, A. J. Liu, and S. R. Nagel, Europhys. Lett. 90, 56001 (2010).

[81] C. F. Schreck, T. Bertrand, C. S. O'Hern, and M. D. Shattuck, Phys. Rev. Lett. 107, 078301 (2011).

[82] H. Yoshino and M. Mézard, Phys. Rev. Lett. 105, 015504 (2010).

[83] G. Szamel and E. Flenner, arXiv:1105.3578 (2011).

[84] A. Donev, S. Torquato, and F. H. Stillinger, Phys. Rev. Lett. 95, 090604 (2005).

[85] L. Berthier, P. Chaudhuri, C. Coulais, O. Dauchot, and P. Sollich, Phys. Rev. Lett. 106, 120601 (2011).

[86] F. H. Stillinger and T. A. Weber, Phys. Rev. B 31, 5262 (1985). 